Mon. Not. R. Astron. Soc. 000, $130(2015) \quad$ Printed 11 October $2017 \quad$ (MN LATEX style file v2.2)

\title{
Multiwavelength observations of nova SMCN 2016-10a - one of the brightest novae ever observed
}

\author{
E. Aydi ${ }^{1,2 \star}$, K. L. Page ${ }^{3}$, N. P. M. Kuin ${ }^{4}$, M. J. Darnley ${ }^{5}$, F. M. Walter ${ }^{6}$, P. Mróz ${ }^{7}$, \\ D. Buckley ${ }^{1}$, S. Mohamed ${ }^{1,2}$, P. Whitelock ${ }^{1,2}$, P. Woudt ${ }^{2}$, S. C. Williams ${ }^{8,5}$, M. Orio ${ }^{9,10}$, \\ R. E. Williams ${ }^{11}$, A. P. Beardmore ${ }^{3}$, J. P. Osborne ${ }^{3}$, A. Kniazev ${ }^{1,12,13}$ \\ V. A. R. M. Ribeiro ${ }^{14,15,16}$, A. Udalski ${ }^{7}$, J. Strader ${ }^{17}$ and L. Chomiuk ${ }^{17}$ \\ ${ }^{1}$ South African Astronomical Observatory, P.O. Box 9, 7935 Observatory, South Africa \\ ${ }^{2}$ Astronomy Department, University of Cape Town, 7701 Rondebosch, South Africa \\ ${ }^{3}$ X-ray and Observational Astronomy Group, Department of Physics \& Astronomy, University of Leicester, LE1 7RH, UK \\ ${ }^{4}$ University College London, Mullard Space Science Laboratory, Holmbury St. Mary, Dorking RH5 6NT, UK \\ ${ }^{5}$ Astrophysics Research Institute, Liverpool John Moores University, IC2 Liverpool Science Park, Liverpool, L3 5RF, UK \\ ${ }^{6}$ Department of Physics and Astronomy, Stony Brook University, Stony Brook NY 11794-3800, USA \\ ${ }^{7}$ Warsaw University Observatory, Al. Ujazdowskie 4, 00-478 Warsaw, Poland \\ ${ }^{8}$ Physics Department, Lancaster University, Lancaster, LA1 $4 Y B$, UK \\ ${ }^{9}$ INAF-Osservatorio di Padova, vicolo dell Osservatorio 5, I-35122 Padova, Italy \\ ${ }^{10}$ Department of Astronomy, University of Wisconsin, 475 N. Charter Str., Madison, WI 53704, USA \\ ${ }^{11}$ Space Telescope Science Institute, 3700 San Martin Drive, Baltimore, MD 21218 USA \\ 12 Southern African Large Telescope Foundation, PO Box 9, 7935 Observatory, Cape Town, South Africa \\ ${ }^{13}$ Special Astrophysical Observatory of RAS, Nizhnij Arkhyz, Karachai-Circassia 369167, Russia \\ ${ }^{14}$ CIDMA, Departamento de Física, Universidade de Aveiro, Campus de Santiago, 3810-193 Aveiro, Portugal \\ ${ }^{15}$ Instituto de Telecomunicações, Campus de Santiago, 3810-193 Aveiro, Portugal \\ ${ }^{16}$ Department of Physics and Astronomy, Botswana International University of Science $\&$ Technology, Private Bag 16, Palapye, Botswana \\ ${ }^{17}$ Center for Data Intensive and Time Domain Astronomy, Department of Physics and Astronomy, Michigan State University, East Lansing, \\ MI 48824, USA
}

Accepted: 2017 October 10 . Received ***; in original form: 2017 July 19

\begin{abstract}
We report on multiwavelength observations of nova SMCN 2016-10a. The present observational set is one of the most comprehensive for any nova in the Small Magellanic Cloud, including: low, medium, and high resolution optical spectroscopy and spectropolarimetry from SALT, FLOYDS, and SOAR; long-term OGLE $V$ - and $I$ - bands photometry dating back to six years before eruption; SMARTS optical and near-IR photometry from $\sim 11$ days until over 280 days post-eruption; Swift satellite X-ray and ultraviolet observations from $\sim 6$ days until 319 days post-eruption. The progenitor system contains a bright disk and a main sequence or a sub-giant secondary. The nova is very fast with $t_{2} \simeq 4.0 \pm 1.0 \mathrm{~d}$ and $t_{3} \simeq 7.8 \pm 2.0 \mathrm{~d}$ in the $V$-band. If the nova is in the SMC, at a distance of $\sim 61 \pm 10 \mathrm{kpc}$, we derive $M_{V \text {, max }} \simeq-10.5 \pm 0.5$, making it the brightest nova ever discovered in the SMC and one of the brightest on record. At day 5 post-eruption the spectral lines show a He/N spectroscopic class and a FWHM of $\sim 3500 \mathrm{~km} \mathrm{~s}^{-1}$ indicating moderately high ejection velocities. The nova entered the nebular phase $\sim 20$ days post-eruption, predicting the imminent super-soft source turn-on in the X-rays, which started $\sim 28$ days post-eruption. The super-soft source properties indicate a white dwarf mass between $1.2 \mathrm{M}_{\odot}$ and $1.3 \mathrm{M}_{\odot}$ in good agreement with the optical conclusions.
\end{abstract}

Key words: stars: individual (SMCN 2016-10a) - novae, cataclysmic variables white dwarfs - ultraviolet: stars - X-rays: binaries. 


\section{INTRODUCTION}

A classical nova $(\mathrm{CN})$ is a result of an eruption on the surface of a white dwarf (WD) in an interacting binary, namely "cataclysmic variable". Classical novae (CNe) consist of an accreting WD and a companion that typically fills its Roche lobe (Warner 1995a). The accreted matter builds up on the surface of the WD and when the pressure and density reach critical levels, a thermonuclear runaway occurs (Payne-Gaposchkin 1964). Within a few days, the eruption increases the visual brightness of the star between 8 and 15 mag. After the peak, the brightness of the star decreases gradually over a timescale of a few weeks up to months. Typically, the ejecta from the eruption can reach a velocity of $\sim 1000 \mathrm{~km} \mathrm{~s}^{-1}$ and contains a mass between $10^{-6} \mathrm{M}_{\odot}$ and $10^{-4} \mathrm{M}_{\odot} \quad$ Gaposchkin 1957, Gallagher \& Starrfield 1978). Although more recently faster, lower ejecta mass systems have been found (Kasliwal et al. 2011; Shara et al. 2017a).

Immediately following the thermonuclear runaway, the surface of the WD is obscured by material thrown out during the eruption. Over time these ejecta expand, becoming optically thin to X-rays, allowing the atmosphere of the nuclear burning region on the WD to become visible. This emission typically peaks in the soft X-ray band, when the nova then enters what is known as the super-soft source (SSS) state (Krautter et al. 1996) (for a review on X-ray observations of CNe see e.g. Osborne 2015 and references therein).

Novae are also observed in ultraviolet (UV) light. In some cases, such as V2491 Cyg (Page et al. 2010) and V745 Sco (Page et al. 2015), the UV light-curve is completely uncorrelated with the X-ray emission, indicating the emission regions are unrelated. HV Cet (Beardmore et al. 2012) is an example where the X-ray and UV emission varies in phase throughout the orbit; in this case, we may be observing a high-inclination (edge-on disk) system, where the X-ray- and UV-emitting region is periodically occulted, perhaps by the lip of an accretion disc. The final situation is one in which the X-rays and UV are anti-correlated for example, V458 Vul (Ness et al. 2009. Schwarz et al. 2011). This could be explained by temperature variations (Schwarz et al. 2011), with the spectral energy distribution shifting between the extreme-UV (EUV) and X-ray bands as the photosphere expands and contracts (see also Schwarz et al. 2015).

Novae are classified based on their optical light-curves and spectra. This classification is determined by the duration in days for the brightness to decrease by 2 or $3 \mathrm{mag}$ from the intensity peak, known as $t_{2}$ and $t_{3}$, respectively (Payne-Gaposchkin 1964, Shafter 1997). Strope, Schaefer \& Henden (2010) classified a sample of 93 nova light-curves based on visual estimates and $V$ band photometry from the AAVSO Photometric All-Sky Survey (APASS 1 - Henden et al. 2009).

From a spectroscopic point of view novae are classified according to the early decline spectra, before they enter the nebular phase (McLaughlin 1944 Gaposchkin 1957.
Williams 2012). Based on the post-eruption spectral features, Williams (1992) divided novae into two spectroscopic classes "Fe II" and "He/N". Aside from the Balmer lines that dominate the spectrum in all classes, the first class exhibits narrow Fe II emission lines with $\mathrm{P}$ Cygni profiles and the second one exhibits broad $\mathrm{He}$ and $\mathrm{N}$ emission lines. Some novae show features from both classes simultaneously or a transition from one class to the other and they are classified as hybrids (Williams 1992, 1994, 2012). The spectra of the Fe II class may be formed in the circumbinary envelope which originated from mass loss from the secondary. The intensity of Fe II spectra fades slowly and lasts for weeks and sometimes months after the eruption due to the low expansion velocities. The spectra of the $\mathrm{He} / \mathrm{N}$ class are thought to originate from the gas on the WD due to the prominence of $\mathrm{He}$ and $\mathrm{N}$ transitions, the rectangular line profiles, and the large line widths (Williams 2012).

Novae in the Milky Way and M31 have been extensively studied with global nova rates of $\sim 34_{-12}^{+15} \mathrm{yr}^{-1}$ and $\sim 65_{-15}^{+16}$ $\mathrm{yr}^{-1}$, respectively (Darnley et al. 2006). In contrast, novae in the Small Magellanic Cloud (SMC) occur less frequently. Since 1897 only 22 novae have been discovered in the SMC (Pietsch 201( [), and most of these novae have been poorly investigated with multiwavelength observations. Little effort has been made to constrain the nova rate for the SMC (see e.g. Graham 1979, Della Valle 2002). Recently Mróz et al. (2016) published an atlas of classical novae in the Magellanic Clouds, offering a systematic study of novae in the SMC where they derived an observed rate of $0.5 \pm 0.2 \mathrm{yr}^{-1}$ and an absolute rate of $0.9 \pm 0.4 \mathrm{yr}^{-1}$. With such a low nova rate, multiwavelength studies of nova events in the SMC are of interest for: (1) understanding better the nova event itself, (2) providing more insights into nova properties in parent galaxies that have different metallicity content, luminosity classes, Hubble types, and star formation histories from the Milky Way or M31 (Mason et al. 2005 Mróz et al. 2016), and (3) the distance modulus to novae belonging to the SMC is known more accurately than is typical for Galactic novae, so that physical properties can also be derived more accurately.

Tracking the evolution of the post-eruption spectral lines and relating this to the multiwavelength light-curve evolution is an important tool to understand the physical mechanism of nova eruptions, hence the importance of extensive low and high resolution spectroscopic monitoring of novae. High resolution spectroscopy allows us to study the evolution of line profiles which provides details about the morphology and velocities of the ejecta, and also offers us the opportunity for modelling the system (Ribeiro, Munari \& Valisa 2013, Ribeiro et al. 2013, De Gennaro Aquino et al. 2015 . Jack et al. 2017).

One of the advantages of studying novae in the Magellanic clouds (MCs) is the known distance (albeit with a finite spread). Deriving distances to novae is a challenge. High ejecta velocities and different light-curve decline rates (e.g. Strope, Schaefer \& Henden 2010) make 


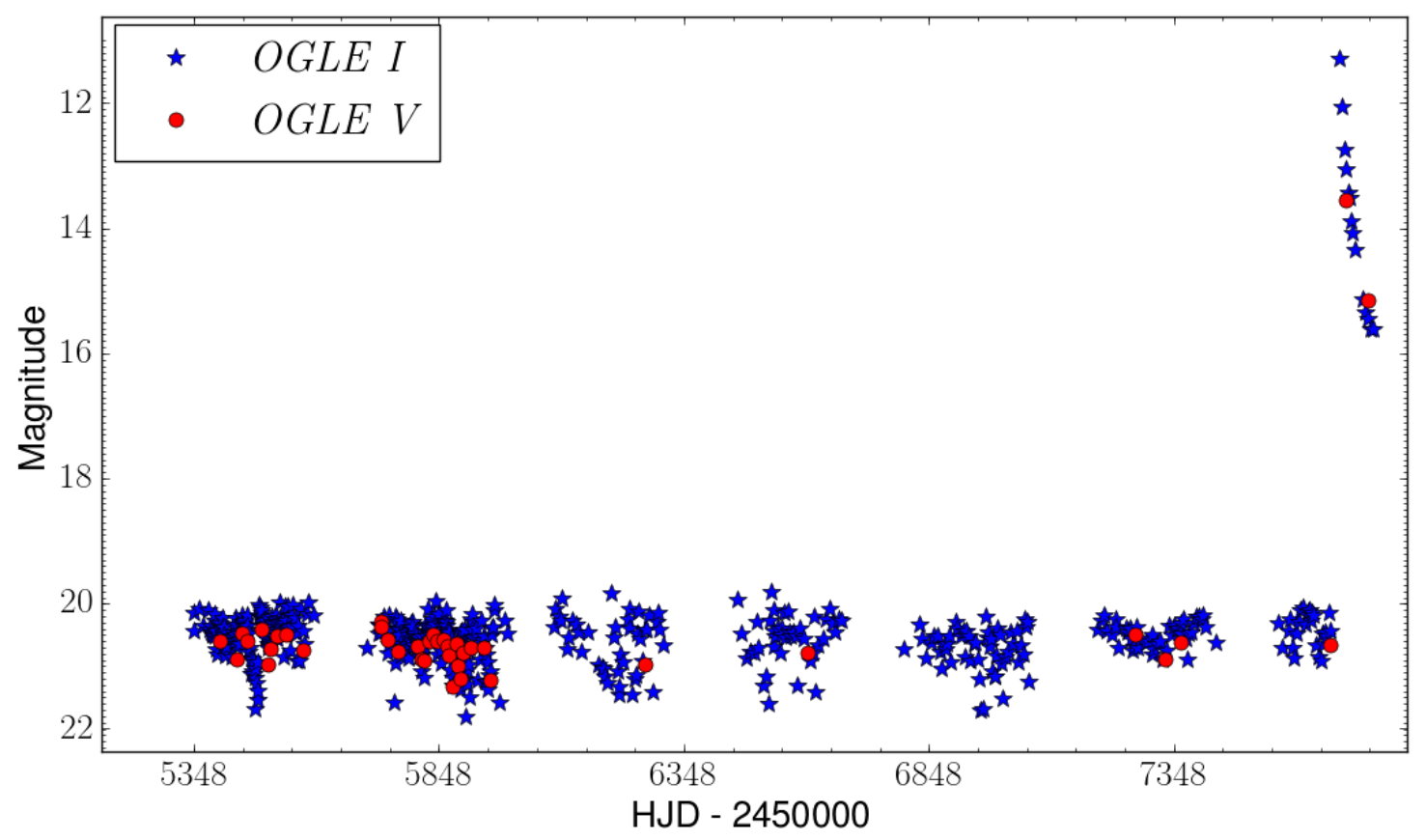

Figure 1. The photometric VI data, from the OGLE-IV Survey (Udalski, Szymański \& Szymański 2015), as a function of Heliocentric Julian Date (HJD), colour and symbol coded as indicated in the legend. The standard error on individual observations in quiescence is less than $0.2 \mathrm{mag}$, so much of the apparent variability is real.

it difficult to derive an accurate estimate. Nevertheless, many methods have been suggested for this purpose. Most of these methods are based on the decline rate of the nova optical light brightness which is correlated with their absolute magnitude at maximum light and are known as "Maximum Magnitude versus Rate of Decline" (MMRD) relations (e.g. McLaughlin 1939. Livio 1992, Della Valle \& Livio 1995, Downes \& Duerbeck 2000). Recently, these methods have been questioned (e.g. Kasliwal et al. 2011. Cao et al. 2012, Shara et al. 2017a). Alternative methods have been proposed to derive distances to novae (see e.g. Downes \& Duerbeck 2000, Shara et al. 2017b) and we will refer to these methods in Section 6.1

In this paper we report on multiwavelength spectroscopic and photometric observations of nova SMCN 201610a that was discovered by MASTER (OT J010603.18744715.8) on HJD 2457675.6 (2016-10-14.2 UT:Shumkov et al. 2016). The eruption started on HJD 2457670.7 (201610-09.2 UT; Jablonski \& Oliveira 2016), this date is considered as $t_{0}$ throughout the paper. The nova is located in the direction of the SMC at equatorial coordinates, $(\alpha, \delta)_{J 2000.0}$ $=\left(01^{\mathrm{h}} 06^{\mathrm{m}} 03^{\mathrm{s}} 27,-74^{\circ} 47^{\prime} 15^{\prime \prime} 8\right)$ (Mroz \& Udalski 2016). It might be the brightest nova ever discovered in the SMC and one of the brightest on record. Multiwavelength follow-up has been carried out to study in detail the post-eruption behaviour of the nova at optical, near-infrared (NIR), X-ray, and UV wavelengths.

The paper is outlined as follows: in Sections 2 and 3 we present and analyze the optical photometric and spectroscopic observations, respectively. We present the X-ray data in Sections 4 and the UV data in Section 5 followed by our discussion in Section 6. We present a summary and the conclusions in Section 7 The observation log is presented in Appendix A

\section{OPTICAL PHOTOMETRIC OBSERVATIONS AND DATA REDUCTION}

\subsection{OGLE observations and data reduction}

The nova has been monitored since 2010 in the $I$ - and $V$-bands by the OGLE-IV Survey (Udalski, Szymański \& Szymański 2015), as part of the Magellanic System survey. These long-term observations provide information on the progenitor system. All data were reduced and calibrated following the standard OGLE pipeline (Udalski, Szymański \& Szymański 2015). Owing to saturation, the data poorly cover the eruption period and the early decline. The last observations before the eruption were made on HJD 2457667.6 (2016 October 6) and the first unsaturated image was obtained on HJD 2457686.6 (2016 October 25). The OGLE $V$ and $I$ - band data are presented in Figure 1

\subsection{SMARTS observations and data reduction}

The Small and Moderate Aperture Research Telescope System (SMARTS) obtained optical BVRI and near-infrared (NIR) JHK photometric observations. The observations started on HJD 2457682.6 (2016 October 21). The integration times at $J H K$ were $15 \mathrm{~s}$ (three $5 \mathrm{~s}$ dithered images) be- 


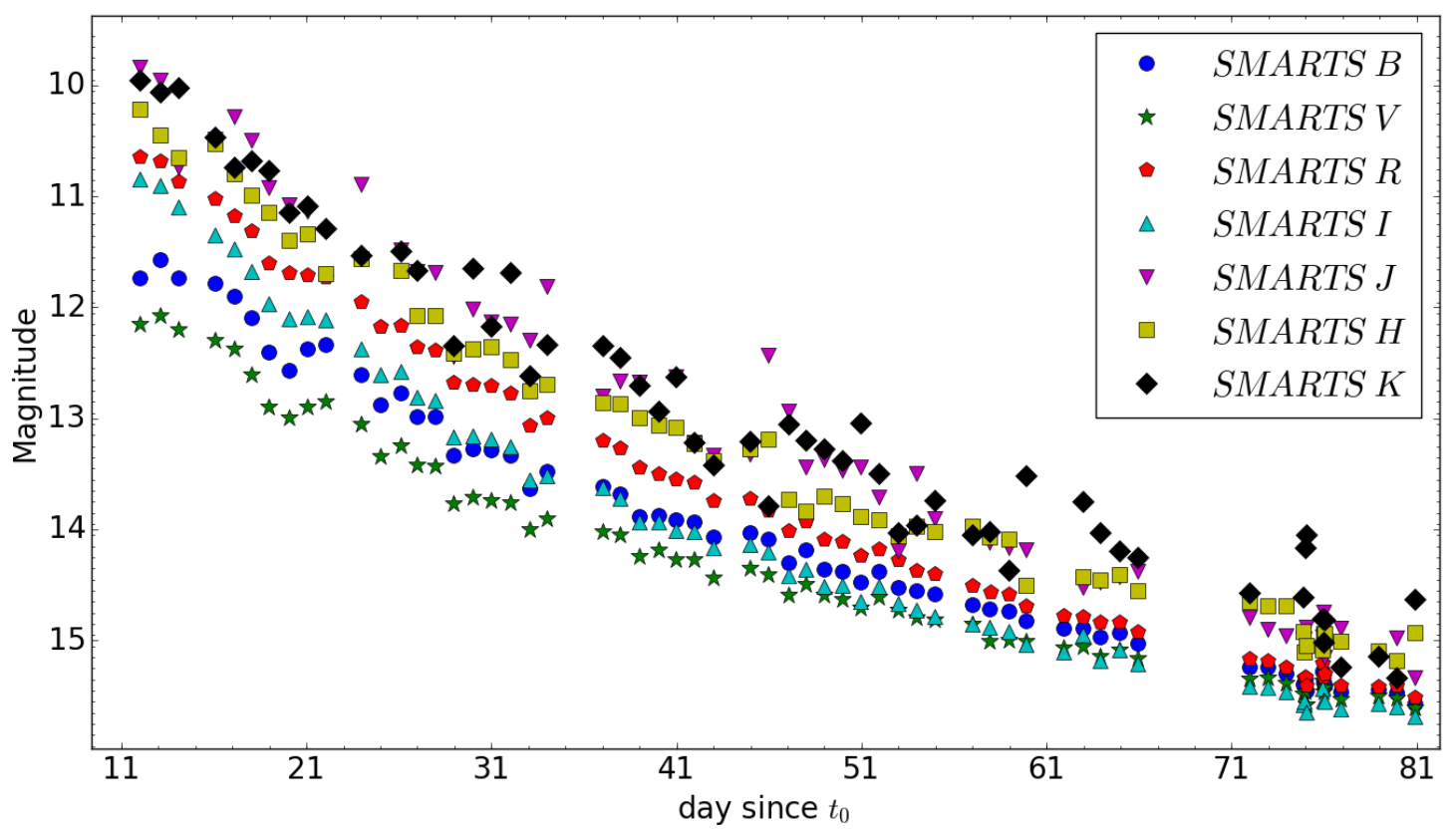

Figure 2. The photometric BVRIJHK data from SMARTS as a function of days since eruption, colour and symbol coded as indicated in the legend. The scatter at $\mathrm{K}$ is due to observational uncertainty. Data after day $\sim 80$ suffer from moderate uncertainties (especially in the $J H K$ bands) and thus are not presented in the plot. A colour version of this plot is present in the online journal.

fore 2016 December 03, and $30 \mathrm{~s}$ thereafter. Optical observations are single images of $30,25,20$, and 20 s integrations respectively in $B V R I$ prior to 2016 December 03, and uniformly $50 \mathrm{~s}$ after 2016 December 03. The details of the data reduction are presented in Walter et al. (2012). The BVRI and $J H K$ data are plotted in Figure 2,

\subsection{Optical light-curve parameters}

CNe light-curves are characterized by several parameters: the rise rate, the rise time to maximum light, the maximum light, the decline rate, and the decline behaviour (morphology) (e.g. Hounsell et al. 2010, Cao et al. 2012). The lightcurves of nova SMCN 2016-10a show a smooth decline with weak oscillations. In comparison to the Strope, Schaefer \& Henden (2010) classification, the light-curves of nova SMCN 2016-10a look like an S-class event. The S-class is considered as the stereotypical nova light-curve and it is characterized by a rapid rise, a relatively sharp peak, followed by a smooth decline that starts off steep and then becomes more gradual with time (Strope, Schaefer \& Henden 2010). Note that the SMARTS optical photometry show a plateau starting about day $\sim 100$ and ending after day $\sim 130$, during solar conjunction (see Figure A1.

On HJD 2457667.67 the nova was observed with OGLE $V$-band at a magnitude of 20.65. Pre-discovery observations by a robotic DSLR camera at Sao Jose dos Campos, Brazil (Jablonski \& Oliveira 2016) showed that on HJD 2457670.7 (2016-10-09.2 UT) the nova was at a magnitude of 12.9. We assume this date as $t_{0}$.

The magnitude then reached 9.9 , after 0.1 days. The nova subsequently brightened to a maximum on HJD
2457671.3 at a magnitude of 8.5. After 1.2 days the magnitude dropped to 8.9, then to 9.3 two days later, indicating the start of the decline. These measurements were obtained using the MASTER Very Wide Field (VWF) camera. According to Gorbovskoy et al. (2010), the instrumental photometric band of the MASTER VWF camera can be described fairly well by the $V_{\mathrm{TYCHO} 2}$ filter and it corresponds to $V$-band photometry (private communication with E. Gorbovskoy). We will consider these measurements as an approximation of $V$-band photometry. Shumkov et al. (2016) discovered the nova at an unfiltered magnitude of 10.9 on HJD 2457675.6.

We consider HJD 2457671.3 (2016-10-9.8 UT) as the day of maximum light and $V_{\max } \approx 8.5$. This means that the magnitude rose from $>13$ to 8.5 in less than 1 day which is relatively fast compared to other classical novae (see e.g. Hounsell et al. 2010, Cao et al. 2012).

In order to get an estimate of the rate of decline, we carried out broken power law and power law fitting to the MASTER and SMARTS magnitudes. We also make use of the All-Sky Automated Survey for Supernovae (ASAS-SN, Shappee et al. 2014) $V$-band measurement $1.7 \mathrm{~h}$ before the discovery. A broken power law is best fitting an S-class lightcurve (see e.g. Hachisu \& Kato 2006, Strope, Schaefer \& Henden 2010). The fitting resulted in $t_{2} \simeq 4.0 \pm 1.0 \mathrm{~d}, t_{3} \simeq$ $7.8 \pm 2.0 \mathrm{~d}$, and a decline rate of $\simeq 0.55 \pm 0.1 \mathrm{mag} \mathrm{d}^{-1}$ over $t_{2}$. A power law index of $\sim 1.76$ was derived from the early slope, in good agreement with the theoretical power law of Hachisu \& Kato (2006) and the empirical values of Strope, Schaefer \& Henden (2010). We also derive $t_{2}$ and $t_{3}$ from a linear interpolation between points and we get very similar results. 


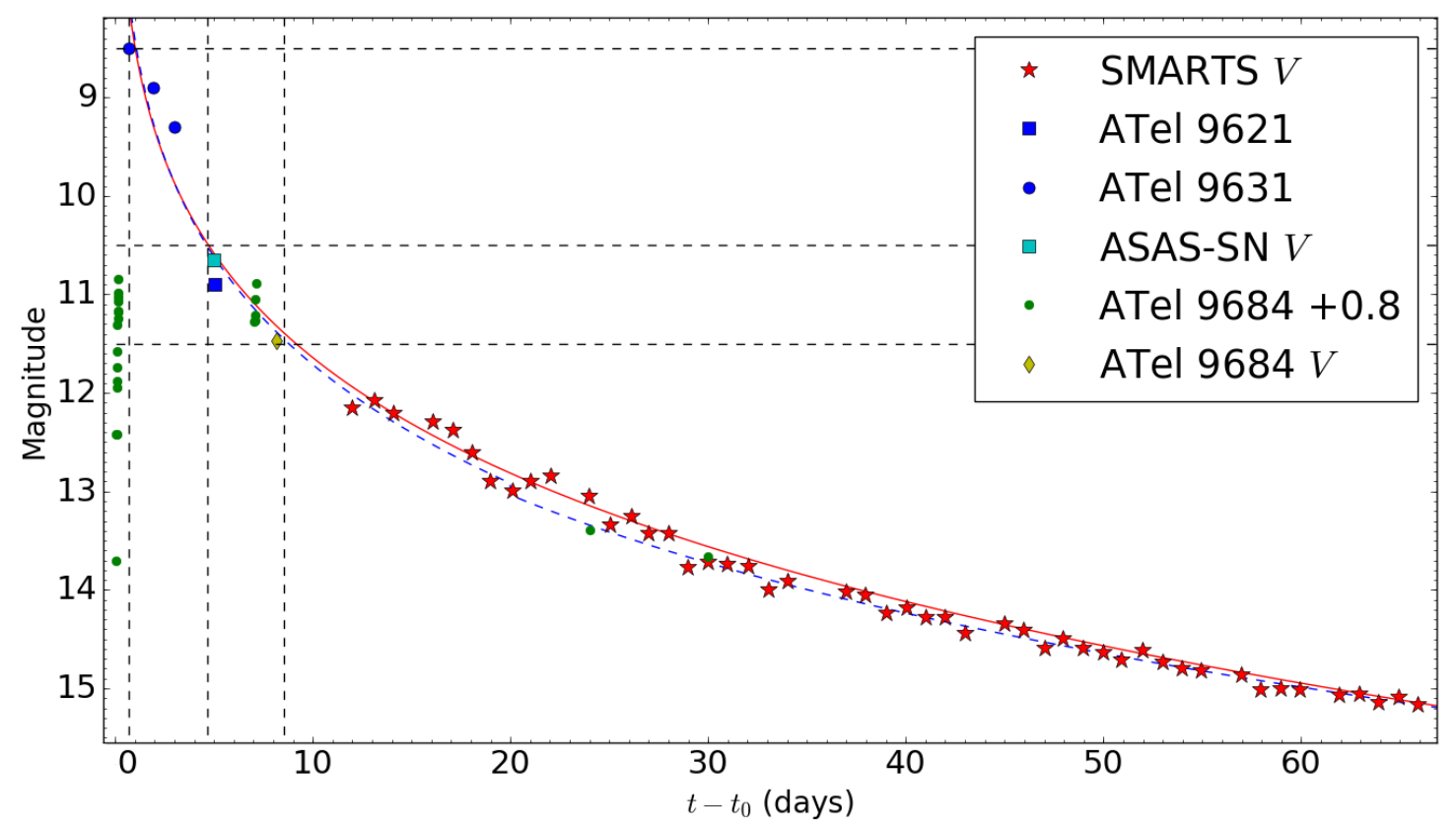

Figure 3. $V$-band photometry from SMARTS and ASAS-SN along with data from Shumkov et al. (2016); Lipunov et al. (2016); Jablonski \& Oliveira (2016). An offset of +0.8 is added to the data from (Jablonski \& Oliveira 2016 see main body of text). The blue dashed line represents a broken power law fit to the data and the red solid line represents a power law fit to the data. The black horizontal dashed lines represent from top to bottom $\left(V_{\max }\right),\left(V_{\max }+2\right)$, and $\left(V_{\max }+3\right)$ respectively. The black vertical dashed lines represent from left to right $t_{\max }, t_{2}$, and $t_{3}$ respectively.

We increased the uncertainty on $t_{2}$ and $t_{3}$ to take into consideration the usage of different measurements from different telescopes and detectors. It is important to recognize that several of our conclusions are dependent on these assumptions. By comparing to the empirical relation from Hachisu \& Kato (2006) which relates $t_{3}$ to $t_{2}$ as: $t_{3}=1.69 t_{2}+0.69 \Delta t_{0}$ days, with the rise time $\Delta t_{0} \sim 0.6$, we obtain $t_{3}=7.1 \pm 1.0$, in agreement with the values estimated above.

In Figure 3 we present the first 70 days of the SMARTS $V$-band photometry along with data from Shumkov et al. (2016); Lipunov et al. (2016); Jablonski \& Oliveira (2016) and the $V$-band measurement from ASAS-SN. The DSLR cameras used to obtain the data from Jablonski \& Oliveira (2016) are very red sensitive and the data require colour correction to be compared with $V$-band photometry. Because of the colour correction required, we exclude these data from the fitting and we just plot them for comparing their agreement with the fit. We adopt an offset of $+0.8 \mathrm{mag}$ from Munari, Hambsch \& Frigo (2017) to correct for the colour difference. As can be seen in Figure 3, the broad DSLR measurement after maximum agree with the simultaneous SMARTS $V$-band data.

Munari, Hambsch \& Frigo (2017) derived different values of $t_{2}$ and $t_{3}$. This is because of the offset they added to the MASTER VWF data (Lipunov et al. 2016). They considered that the VWF is very red sensitive and they added an offset of $+1 \mathrm{mag}$ to correct for the colour difference, leading them to larger values of $t_{2}, t_{3}$, and $V_{\max }$. Since the MASTER VWF measurements correspond to $V$-band photome- try (Gorbovskoy, private communication), such an offset is too large.

Payne-Gaposchkin (1964) classified the light-curves of novae into 5 speed classes. Novae with $t_{2}<10$ days and $\dot{m}_{v}>0.20 \mathrm{mag} \mathrm{d}^{-1}$, which is the case for this nova, are classified as "very fast".

\section{OPTICAL SPECTROSCOPIC OBSERVATIONS AND DATA REDUCTION}

\subsection{SALT medium resolution spectroscopy}

We observed the nova on 2016 October 17 (day 8) and November 1 (day 23) using the Robert Stobie Spectrograph (RSS; Burgh et al. 2003 Kobulnicky et al.2003), in Long Slit (LS) mode, mounted on the Southern African Large Telescope (SALT; Buckley, Swart \& Meiring 2006, O'Donoghue et al. 2006) situated at the SAAO, Sutherland, South Africa. Both observations consist of two spectral ranges; [3500 $\AA$ $6300 \AA]$ and $[6000 \AA-9000 \AA]$. For the October 17 observations the RSS long-slit mode was used, with a 1.5 arcsec slit and the PG900 grating, resulting in a resolution of $R \sim 850$ for the first range and $R \sim 1400$ for the second one. For the November 1 observations we used a 1.0 arcsec slit and the PG900 grating, resulting in a resolution of $R \sim 1250$ for the first range and $R \sim 2000$ for the second one. For both observations, ThAr and Ne lamp arc spectra were taken immediately after the science frames for the first and second range respectively.

The spectra were first reduced using the PySALT 

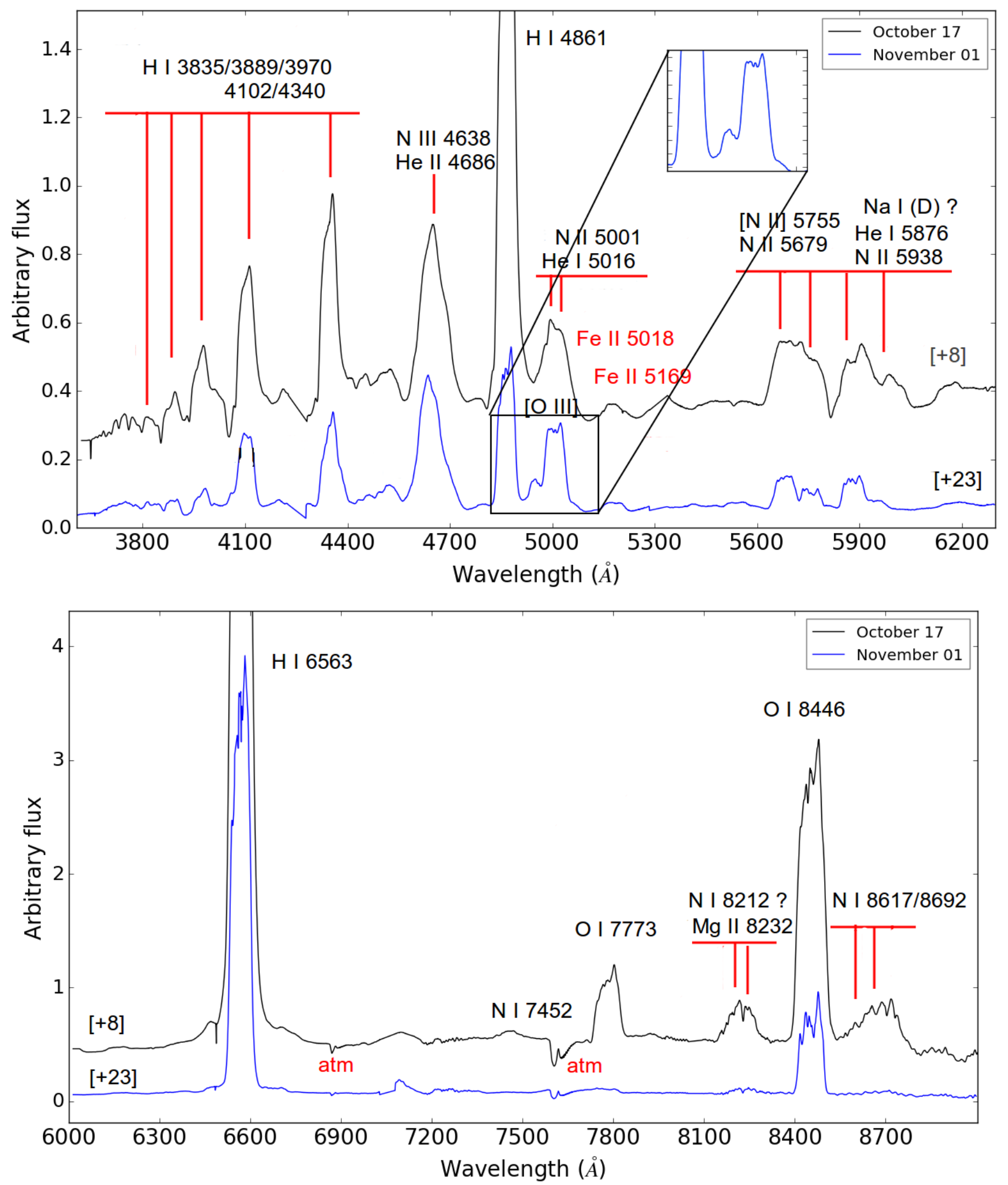

Figure 4. The LS medium resolution spectra plotted between $3600-6300 \AA$ and $6000-9000 \AA$ with the flux in arbitrary units. For clarity, the spectra are shifted vertically. The numbers between brackets are days since $t_{0}$. 
pipeline (Crawford et al. 2010), which involves bias subtraction, cross-talk correction, scattered light removal, bad pixel masking, and flat-fielding. The wavelength calibration, background subtraction, and spectral extraction are done using the IRAF (Image Reduction and Analysis Facility) software (Tody 1986). The spectra are presented in Figure 4

\subsection{SALT high resolution echelle spectroscopy}

The SALT High Resolution Spectrograph (HRS; Barnes et al. 2008, Bramall et al. 2010, Bramall et al. 2012, Crause et al. 2014), a dual-beam, fibre-fed echelle spectrograph, was used to obtain high resolution observations on the nights of 2016 October 17, November 5, November 10, November 23, and December 10 (respectively day 8, 27, 32, 45, and 65). The observations were taken in the low-resolution (LR) mode of HRS to provide a spectrum covering a spectral range of $3800 \AA-9000 \AA$ at a resolution of $R \sim 15000$. A weekly set of HRS calibrations, including four ThAr $+\mathrm{Ar}$ arc spectra and four spectral flats, was obtained in all the modes (low, medium, and high resolution).

Primary reduction of the HRS data was done using the SALT science pipeline (Crawford et al. 2010), which includes over-scan correction, bias subtraction, and gain correction. The rest of the reduction was done using the standard MIDAS FEROS (Stahl, Kaufer \& Tubbesing 1999) and echelle (Ballester 1992) packages. The reduction procedure is described in detail in Kniazev, Gvaramadze \& Berdnikov (2016). The spectra are presented in Figure 5

\subsection{FLOYDS spectroscopy}

A series of eleven epochs of spectroscopy of nova SMCN 2016-10a were obtained on the nights of 2016 October 14, $17,18,19,20,22,23,29,31$, November 02, and 10 (respectively day $5,8,9,10,11,13,14,20,22,24,32)$ using the FLOYDS spectrograph ${ }^{3}$ on the Las Cumbres Observatory (LCO; Brown et al. 2013) 2.0m telescope at Siding Springs, Australia 4 FLOYDS is a cross-dispersed low resolution $(R \sim 550)$ spectrograph using an Andor Newton DU940P-BU detector and with a wavelength coverage of $3200 \AA-10000 \AA$. Data reduction was carried out using the FLOYDS data pipeline (v2.2.1). See Table A3 for a log of the observations. The flux calibrated spectra are presented in Figure 6. Due to poor photometric conditions during the observations as well as slit losses, the absolute flux calibration is not to trust as photometric data.

\subsection{SOAR spectroscopy}

We also performed spectroscopy of the nova on 2017 July 11, using the Goodman spectrograph (Clemens, Crain \& Anderson 2004) on the Southern Astrophysical Research (SOAR) telescope. One single $1200 \mathrm{~s}$ spectrum was obtained, using a $400 \mathrm{l} \mathrm{mm}^{-1}$ grating and a $1.07^{\prime \prime}$ slit, giving resolution of $\sim 7 \AA$ over the wavelength range $3810 \AA-7860 \AA$. The spectrum was reduced in the standard manner, with a relative

\footnotetext{
3 https://lco.global/observatory/instruments/floyds
}

4 Formally known as Faulkes 'Telescope South. flux calibration applied, and including an approximate correction for slit losses. The spectrum is presented in Figure 7

\subsection{Line identification}

The strongest features in the spectra of day 5 and 8 (Figure 4, 5, and 6) are the broad Balmer lines $(\mathrm{H} \alpha, \mathrm{H} \beta, \mathrm{H} \gamma$, $\mathrm{H} \delta, \mathrm{H} \epsilon, \mathrm{H} \zeta$, and $\mathrm{H} \eta$ ). The hydrogen Balmer lines are accompanied by weak absorption features. The radial velocities (RVs) derived from the P Cygni absorption on day 8 , by measuring the wavelengths at the minimum flux, are as follows: $\mathrm{H} \gamma \sim-2300 \pm 200 \mathrm{~km} \mathrm{~s}^{-1}, \mathrm{H} \delta \sim-2700 \pm 200 \mathrm{~km} \mathrm{~s}^{-1}$, $\mathrm{H} \epsilon \sim-2700 \pm 200 \mathrm{~km} \mathrm{~s}^{-1}$, and $\mathrm{H} \zeta \sim-2500 \pm 200 \mathrm{~km} \mathrm{~s}^{-1}$. Another set of prominent features are the Oxygen lines (O I 7773 and $8446 \AA$ ). Nitrogen emission lines are also present (N III $4638 \AA$, N II $5001 \AA$, $5679 \AA$, $5938 \AA$, [N II] $5755 \AA$, N I $7452 \AA, 8212 \AA, 8617 \AA$, and $8692 \AA$ ), noting that the N III $4638 \AA$ might be accompanied by a weak P Cygni absorption. There is a possible weak detection of He I $7065 \AA$. He II $4686 \AA$ and He I $5016 \AA$ might be blended with their neighbouring nitrogen lines. He I $5876 \AA$ might also be affected by $\mathrm{Na}$ I D and the nitrogen line at $5938 \AA$. Mg II $7896 \AA$ and $8232 \AA$ are also present. The line at $5169 \AA$ is possibly Fe II while the other multiplet (42) lines at $4924 \AA$ and $5018 \AA$ might be blended with other lines.

The spectra from day 9 to 14 show the same emission features with the O I $7773 \AA$ and N II $5001 \AA$ lines fading gradually. The spectra of day 20 to 23 (Figure 4 and 6 ) show similar lines, with the appearance of [O III] $4959 \mathrm{~A}$ and $5007 \AA$ nebular lines. The weak absorption features had faded. The O I $7773 \AA$ line weakened compared to the $8446 \AA$ line. The [N II] $6548 \AA, 6583 \AA$ might be blended with the broad $\mathrm{H} \alpha$ line (see section 6.6.1).

In the spectra of day 32 (Figure 6) forbidden Ne lines emerge at $3346 \AA$ and $3426 \AA$ (see Section 5.2), while the Balmer features start to fade compared to the [O III] emission lines (4363 $\AA, 4959 \AA, 5007 \AA)$. The O I lines at $7773 \AA$ and $8446 \AA$ further in the red also faded noticeably. The spectra of day 45 and 65 show strong emission lines of [O III] and He I $4388 \AA$ in the blue while the O IV $7713 \AA$ and C IV $7726 \AA$ nebular lines emerge.

The late spectra of day 275 (Figure 7) were dominated by the [O III] $4363 \AA, 4959 \AA$, and $5007 \AA$ lines, along with $\mathrm{H} \alpha$. The spectral line identification was primarily done using the list from Williams $(2012)$ and the Multiplet Table of Astrophysical Interest (Moore 1945). In Table A9 we list the line IDs along with the FWHM, Equivalent Widths (EWs), and fluxes for those emission lines for which an estimate was possible.

\subsection{SALT spectropolarimetry}

Spectropolarimetry observations were obtained on the nights of 2016 October 24 and November 21 (days 15 and 43) using the RSS spectrograph. The RSS is capable of imaging polarimetry and spectropolarimetry (for more details on the optics see Nordsieck et al. 2003). Both observations consist of two spectral ranges; $[3500 \AA-$ $6300 \AA]$ and $[6000 \AA-9000 \AA]$. The RSS LS mode was used, with a 1.5 arcsec slit and the PG900 grating, resulting in 

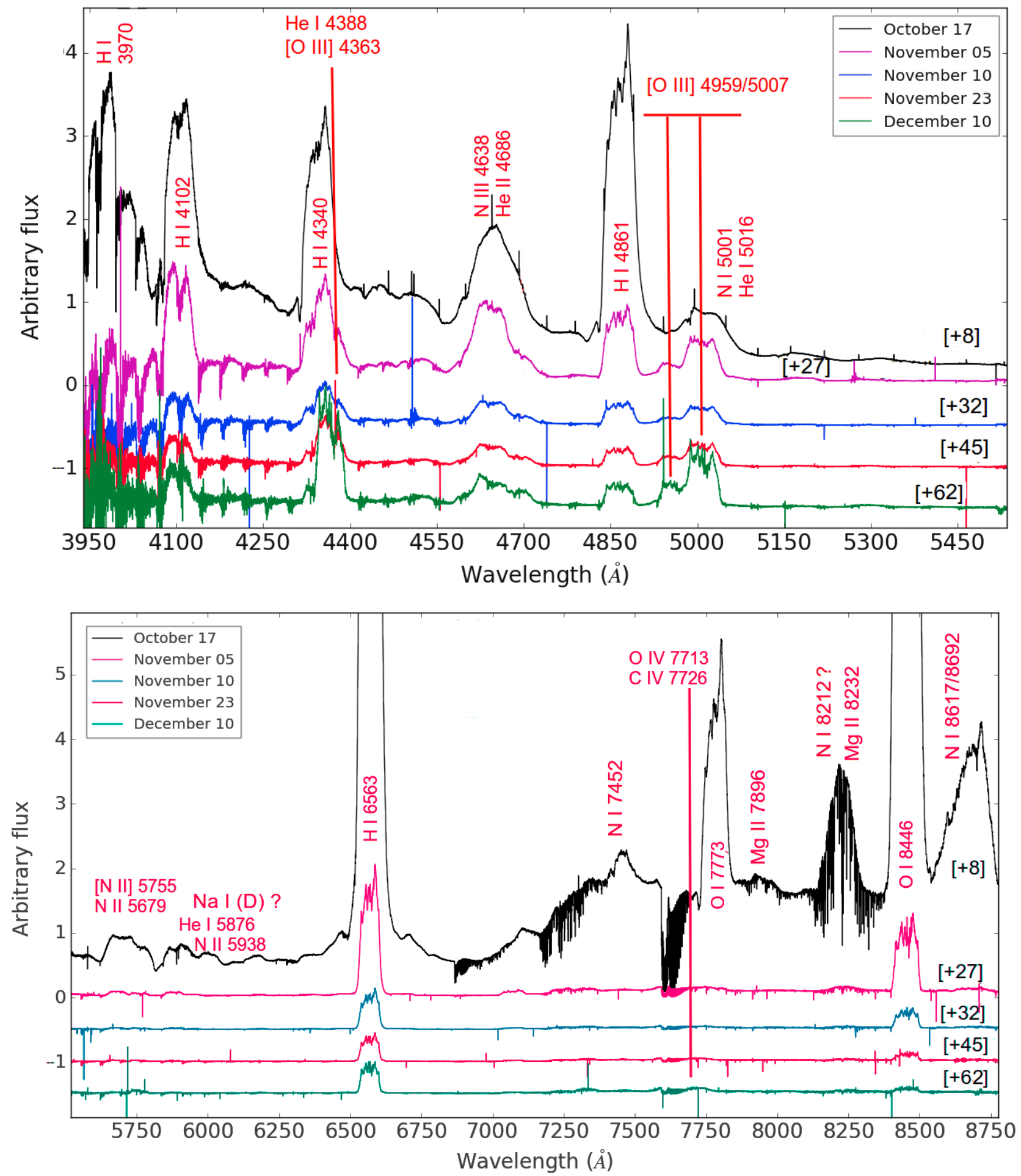

Figure 5. The HRS high resolution spectra plotted between $3900-5500 \AA$ and $5700-8750 \AA$ with the flux in arbitrary units. For clarity, the spectra are shifted vertically. The numbers between brackets are days since $t_{0}$.

a resolution of $R \sim 850$ for the first range and $R \sim 1400$ for the second one. ThAr and Ne lamp arc spectra were taken immediately before the science frames for the first and second range, respectively.

The spectropolarimetry data reduction was carried out using the PolSALT pipeline (Crawford et al. in preparation). This pipeline performs basic reductions (e.g. bias subtraction, cross-talk correction, over-scan correction, and cosmic-ray rejections), wavelength calibration and spectrum extraction, whereafter the Stokes parameters are 

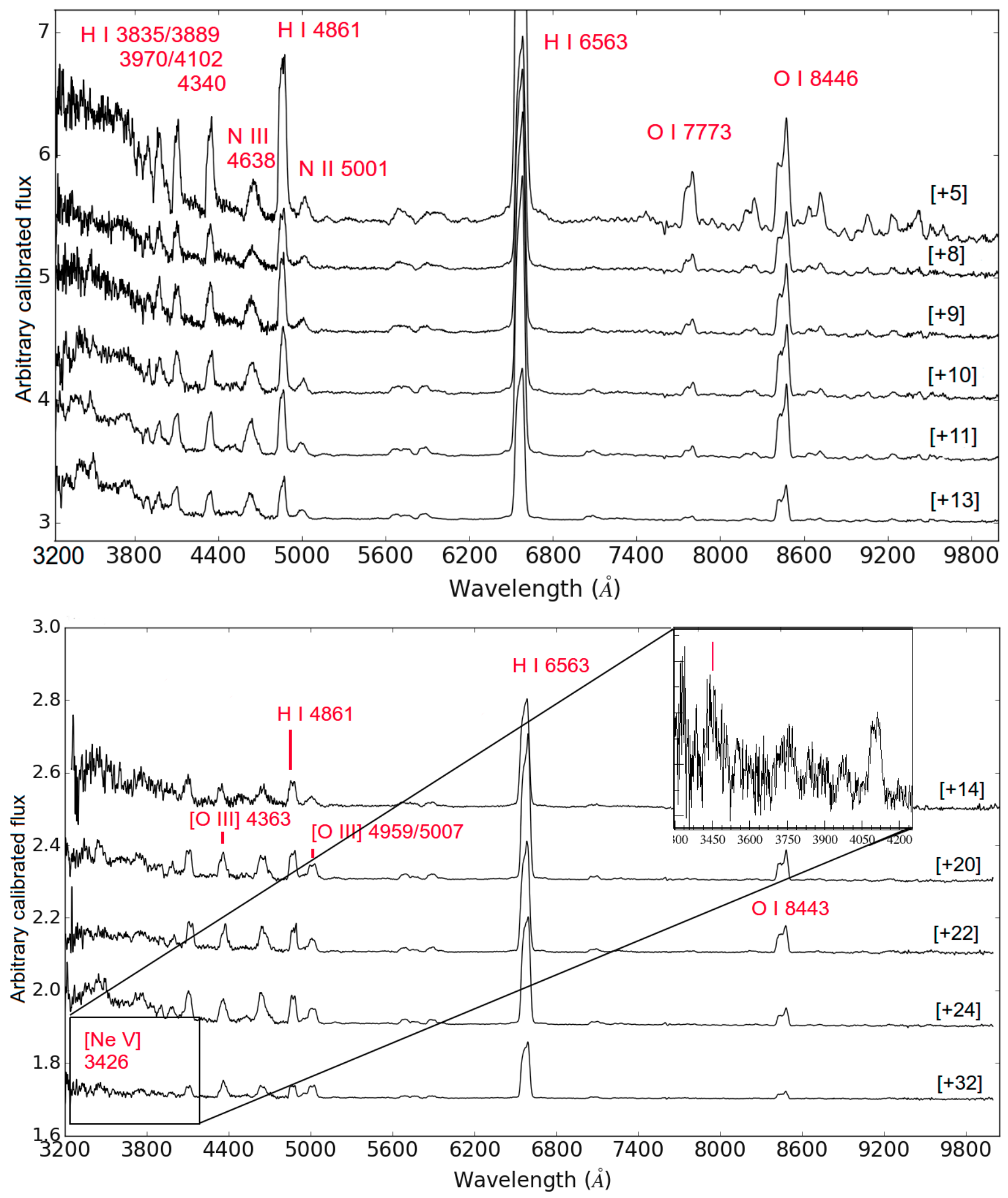

Figure 6. The FLOYDS spectra plotted between $3200-10000 \AA$, with the calibrated flux in arbitrary units. For clarity, the spectra are shifted vertically. From top to bottom: Oct 14, 17, 18, 19, 20, 22, 23, 29, and 31, Nov 02, and 10. The numbers between brackets are days since $t_{0}$. 


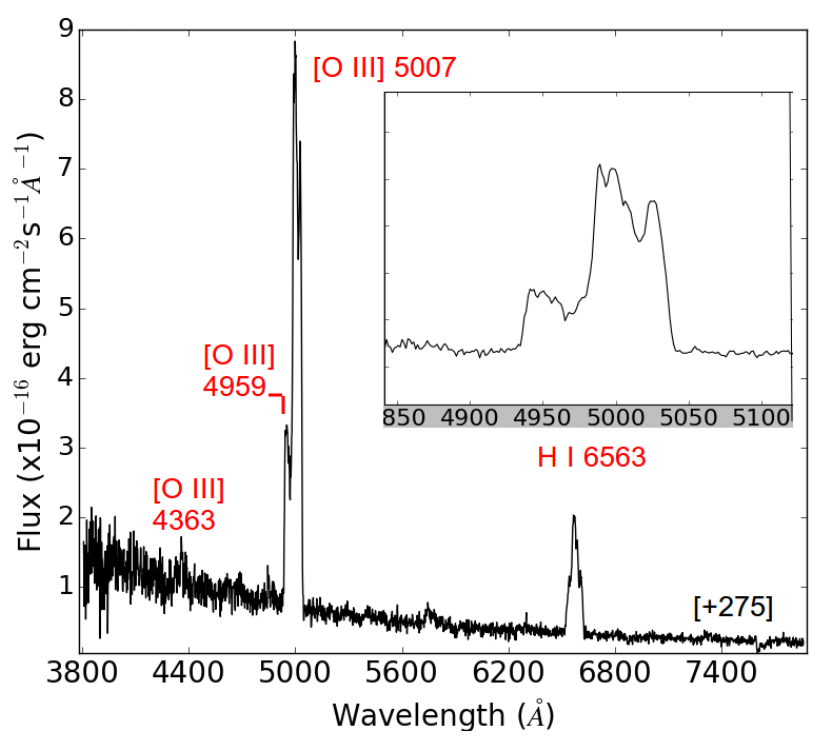

Figure 7. The SOAR spectrum of day 275 plotted between $3800 \AA-7860 \AA$, with the calibrated flux in $\left(\operatorname{erg~cm} \mathrm{cm}^{-2} \mathrm{~s}^{-1} \AA^{-1}\right)$. The number between brackets is days since $t_{0}$. We added a zoomin plot on the [O III] $4959 \AA$ and $5007 \AA$ lines for clarity.

determined.

The linear polarization, and the position angle are plotted as a function of wavelength in Figure 8 . We show the observations from day 15 as a sample for both observations. The enhancement at the blue and red ends of the spectra might be due to low signal to noise ratio and hence we consider only the range between $4000 \AA$ and $8000 \AA$ in our analysis. The linear polarization is on average $\sim 0.3 \%$. This may be due to the interstellar medium or intrinsic to the nova, or both. Rodrigues et al. (1997) studied the interstellar polarization toward the SMC. Nova SMCN 2016-10a is situated just south of their region III which has the smallest foreground polarization $(P=0.17 \%)$.

In order to estimate the contribution of the interstellar medium, we derive the linear polarization at different wavelengths using the Serkowski empirical relation (Serkowski, Mathewson \& Ford 1975, Wilking et al. 1980), and assuming $p_{\max }=0.4 \%$ at $\lambda=4450 \AA$. The values are plotted in Figure 8 . The polarization is below the values derived for the interstellar medium.

Although, the interstellar polarization is low, it is nevertheless likely to be the primary source of polarization in our observations. There is no sign of de-polarization effect due to emission lines that is usually observed in case of polarization originating from novae (see e.g. Ikeda, Kawabata \& Akitaya 2000). The polarization in novae can originate either from scattering on dust or from a magnetic field, so if the observed polarization is indeed from the interstellar medium, the lack of intrinsic polarization can indicate the following: First, that there is no dust in the ejecta, consistent with the behaviour of the optical and infrared light-curves. Secondly, there might be no or weak magnetic field.

\section{X-RAY OBSERVATIONS}

Swift (Gehrels et al. 2004) observations of nova SMCN 201610a started on 2016 October 15 (6.1 days after $\left.t_{0}\right)$. At this time, no X-rays were detected, but a $500 \mathrm{~s}$ grism spectrum showed a bright UV source with strong lines (Kuin et al. 2016). Additional observations were performed on the following two days, after which the cadence was lowered to once every few days. On 2016 November 07 (day 28.2), a bright, soft X-ray source was detected, at a count rate of $1.57 \pm 0.06$ count $^{-1}$. In order to parameterize the onset of the SSS phase, multiple short snapshots of data were collected on 2016 November 11-12 (days 33-34), revealing the high amplitude flux variability seen in a number of other novae (e.g. Ness et al. 2009, Osborne et al. 2011, Bode et al. 2016). Observations then continued approximately every two to four days until 2017 May 12, with further observations following an eight day cadance running until 2017 June 25. Five further observations were obtained between 2017 July 8 and August 24. We note that the location of the SMC is not observable by Swift for up to 10 days each month because of the so-called "pole constraint"; this arises from the requirement that Swift not point too close to the Earth limb.

The Swift data were processed with HEASoft 6.19, and analyzed with the most up-to-date calibration files. The Xray Telescope (XRT; Burrows et al. 2005) was operated in "Auto" state, so that the most appropriate mode (Windowed Timing - WT - when the source was above about 5 count $^{-1}$; Photon Counting - PC - when the source was fainter) would be automatically chosen. Reaching a peak count rate of $\sim 50$ count $^{-1}$, the WT data did not suffer from pile-up, so a circular extraction region was used for these data. PC mode data above around 0.5 count $\mathrm{s}^{-1}$ were considered to be affected by pile-up, so, at these times, the core of the Point Spread Function was excluded, and corrected for, as required, using annular extraction regions. Background counts were estimated from nearby, source-free regions. In order to facilitate Cash statistic (Cash 1979) fitting within XSPEC (Arnaud 1996), the X-ray spectra were binned to ensure a minimum of 1 count bin ${ }^{-1}$. Fitting using the C-statistic (modified by XSPEC to work in the case of a background spectrum) provides less biased parameter estimates, even in the high-count regime (Humphrey, Liu \& Buote 2009).

The first X-ray detection of nova SMCN 2016-10a showed the emission already to be super-soft. At the distance of the SMC, any underlying shock emission would be likely to be faint and difficult to detect (cf. Nova LMC 2009a; Bode et al. 2016). Assuming optically thin emission at a temperature of $5 \mathrm{keV}$, and combining all the X-ray data collected, a $90 \%$ upper limit of $\sim 2 \times 10^{34} \mathrm{erg} \mathrm{s}^{-1}$ can be placed on the unabsorbed 0.3-10 keV luminosity for any hard spectral component. The X-ray spectra were therefore fitted below $1 \mathrm{keV}$ with an absorbed plane-parallel, static, non-local-thermalequilibrium stellar atmosphere model (grid 003) $)^{5}$ samples of the spectra are shown in Figure 9 , and the parameter results from the fitting are shown in Figure 10 while Figure 11 focuses in on the early variability phase. This highlights the

$\begin{array}{lcrr}5 \text { TMAP: } & \text { Tübingen } & \text { NLTE } & \text { Model At- } \\ \text { mosphere } & \text { Package: } & \text { http://astro.uni- } \\ \text { tuebingen.de/ } \sim \text { rauch/TMAF/flux_HHeCNONeMgSiS_gen.html }\end{array}$




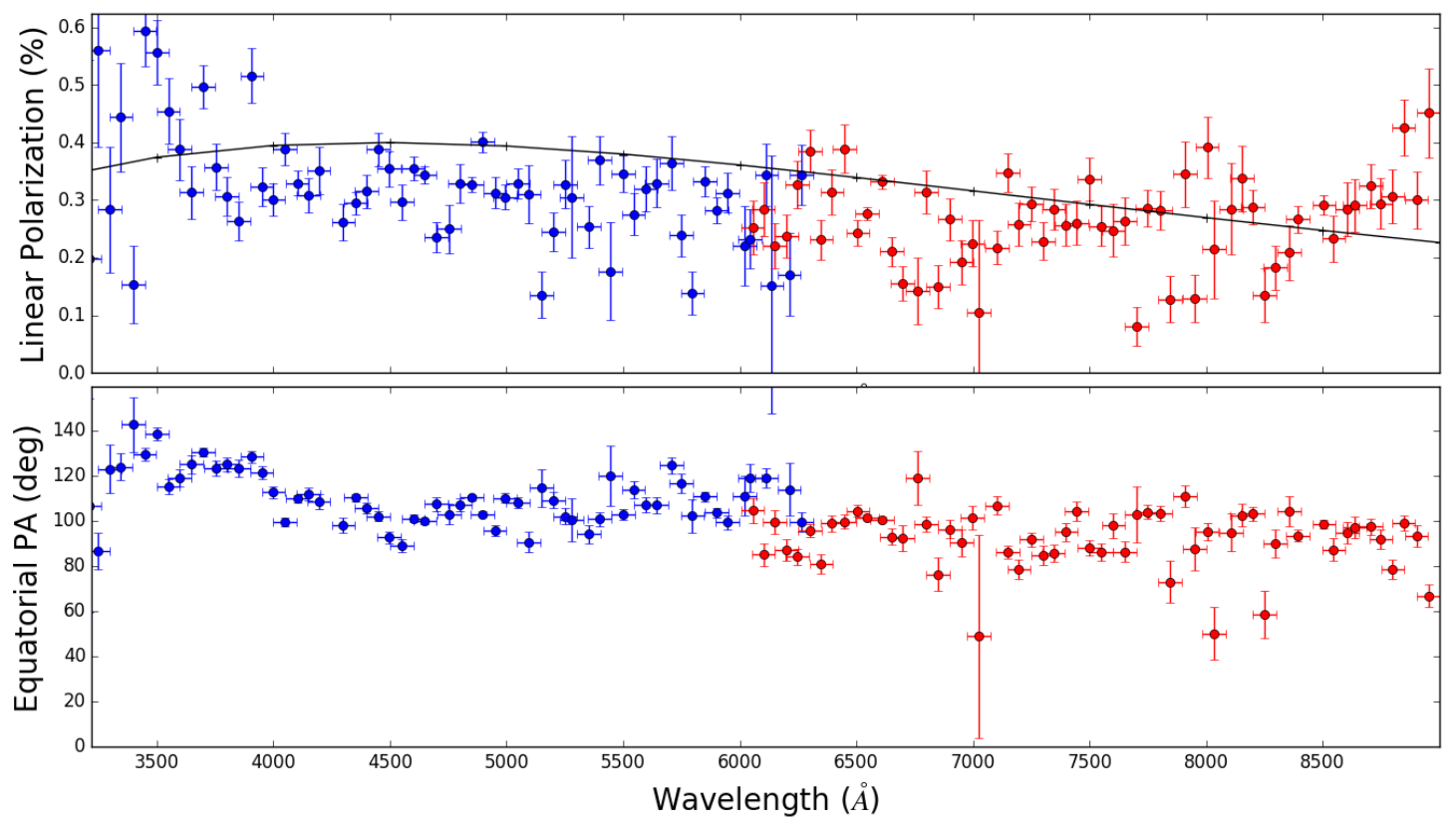

Figure 8. The linear polarization (top) and position angle (bottom) plotted against the wavelength from day 15 . The blue and red points correspond to observations from the first and second spectral range respectively. The black solid line represents the values derived from the Serkowski relation, assuming $p_{\max }=0.4 \%$ at $\lambda=4450 \AA$, for the polarization due to the interstellar medium. A colour version of this plot is present in the online journal.

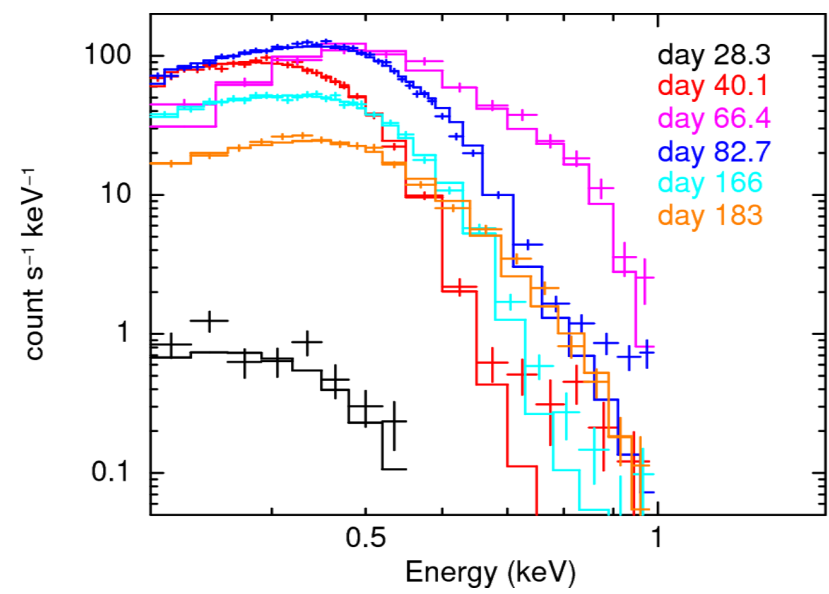

Figure 9. A sample of Swift X-ray spectra from days 28.3, 40.1, $66.4,82.7,166$ and 183 since eruption $\left(t_{0}\right)$, fitted in each case with the best-fit TMAP atmosphere model (see Figure 10. A colour version of this plot is present in the online journal.

order of magnitude or more variability seen in the $\mathrm{X}$ ray count rate over an interval of two days as the SSS became established. The atmosphere grids typically provide good fits, with the reduced Cash statistic values mainly lying in the range 0.8-1.2. The model atmosphere is limited to temperatures of between $\sim 38 \mathrm{eV}$ and $91 \mathrm{eV}$, and a small number of the spectra for this nova had temperatures which hit the upper or lower limits of these bounds, suggesting the actual values are outside the range covered by the grids; these are shown by upper or lower limits in the second panel of
Figure 10 and Figure 11 as necessary. The third panel of Figure 10 and 11 show the bolometric luminosity of the soft emission component, in terms of the Eddington luminosity for a $1.2 \mathrm{M}_{\odot} \mathrm{WD}$, assuming a distance of $61 \mathrm{kpc}$. The absorbing column was allowed to vary in the fits, and is shown in the bottom panel of Figure 10 and 11

The temperature of the SSS was lower at earlier times, increasing suddenly after day 60, and reaching an apparent peak (as shown by the magenta spectrum in Figure 9) at a time the source location unfortunately became nonobservable by Swift. Following the emergence from the observing constraint (day 82.7), the temperature of the SSS emission was consistently high, $\sim 90 \mathrm{eV}$. We note that there are occasional fast changes in the X-ray count rate, for example around day 36.5, without corresponding variations in the spectral shape (i.e. the temperature remains approximately constant). This implies that there must be partial obscuration by clumps of high optical depth material at these times.

Even as the X-ray emission started to fade sharply, after day 180 , the temperature remained high, actually increasing slightly compared to earlier spectra. However, on day 237 a significant drop in temperature was observed. A final cooling of the X-ray emission is expected (Soraisam et al. 2016), and has been seen in other novae well-monitored by Swift (Page \& Osborne 2014). From day 242 onwards, the X-ray spectrum was not well fitted by the atmosphere model, with the shape better parametrized by an optically thin component with $\mathrm{kT} \sim 80 \mathrm{eV}$. These data-sets have therefore not been included in panels 2-4 in Figure 10 This adds to the evidence that the eruption has ended. As the X-rays faded, there was no longer any evidence for excess absorption above 


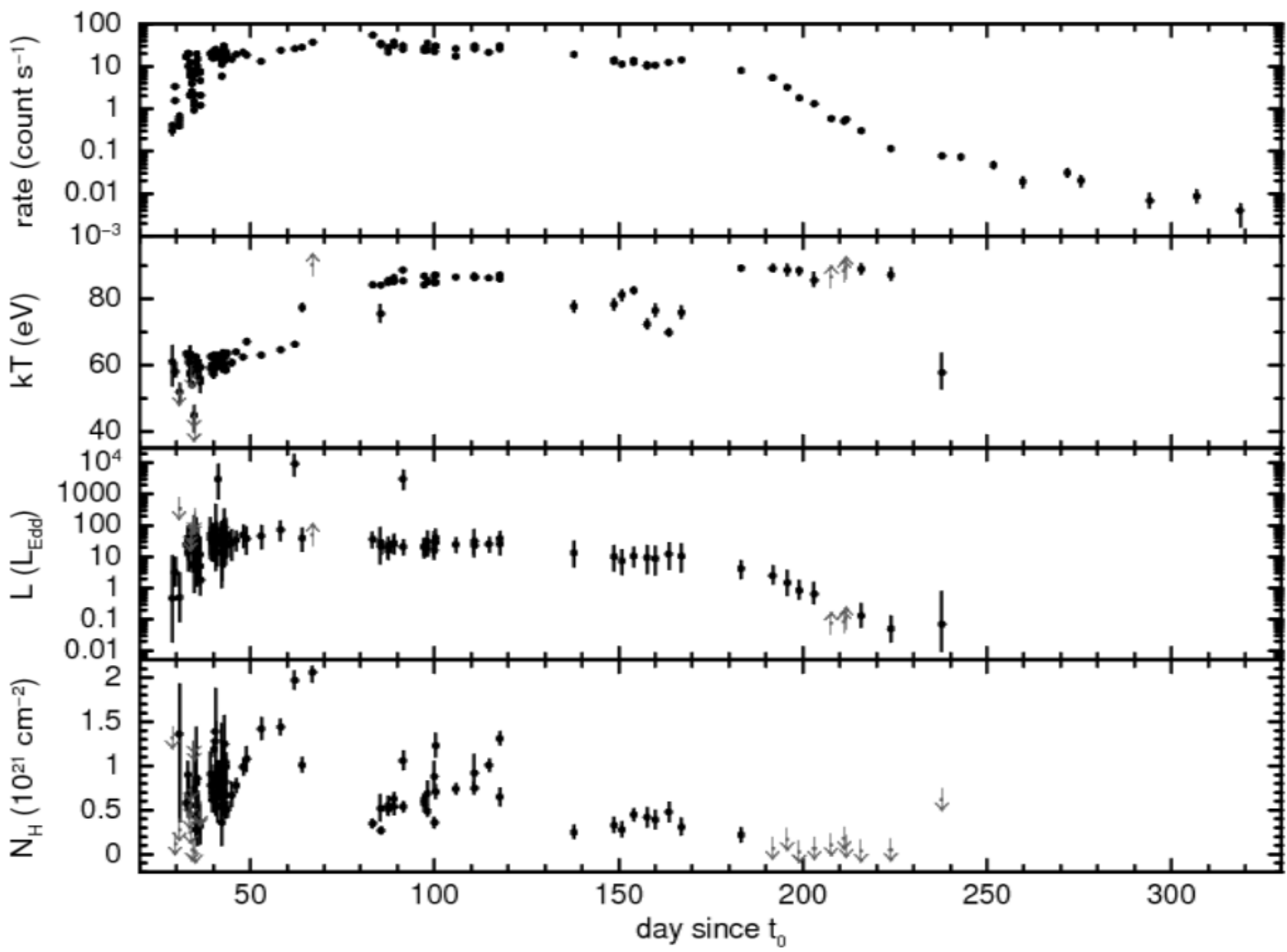

Figure 10. From top to bottom: the count rate, the temperature of the SSS emission, the bolometric luminosity in units of the Eddington value for a $1.2 \mathrm{M}_{\odot} \mathrm{WD}$, and the absorbing column density plotted against the days since $t_{0}$ (HJD 2457670.70). All parameters were determined from the TMAP model atmosphere fits as described in the text. Grey arrows indicate upper or lower limits (90\%) as necessary. For the luminosity calculation, a distance $=61 \mathrm{kpc}$ is used.

the interstellar value in the direction of the SMC $\left(6 \times 10^{20}\right.$ $\mathrm{cm}^{-2}$; Coe et al. 2011); in fact, some of the late-time upper limits suggest that the total absorbing column is below this value suggesting either an uncertainty in the absorption in the direction of the SMC, or a limitation of the emission model applied to the X-ray spectra.

Krautter et al. (1996) first showed that the X-ray emission from a fading post-eruption nova remains soft. The residual material is hot and extended and, even as the nuclear fusion comes to an end, the evolving WD decreases in radius (towards the quiescent radius of the star) at nearly the same luminosity for a while, leading to an increase in temperature.

A number of novae with bright $\mathrm{X}$-ray emission have shown short period $(<100 \mathrm{~s})$ oscillations (e.g. Osborne et al. 2011; Beardmore et al. 2010, Ness et al. 2015). We have therefore searched for the presence of such oscillations in the soft X-ray light-curves of nova SMCN 2016-10a, following the procedure outlined in Beardmore et. al. (in prep.).

WT light-curves were extracted with a time bin size of $17.8 \mathrm{~ms}$ over the energy range $0.3-1.0 \mathrm{keV}$ (i.e. where the SSS emission is dominant). Periodograms were then computed from continuous light-curve sections up to $512 \mathrm{~s}$ duration given the short nature of some of the XRT snapshots, those with exposures less than $256 \mathrm{~s}$ were rejected while those between $256-512 \mathrm{~s}$ were padded to a duration of $512 \mathrm{~s}$ using the mean rate for the interval. The average periodogram constructed from 80 individual ones spanning days 32.03 to 157.07 reveals no evidence for any oscillations with a timescale ranging from $1-256 \mathrm{~s}$, with a 3 sigma fractional rms upper limit of 1.4 per cent.

\section{UV OBSERVATIONS}

\subsection{Swift UVOT photometry}

UV/Optical Telescope (UVOT; Roming et al. 2005) photometric observations started on day 12 (2016 October 21) and continued until day 319 (2017 August 24). The initial photometry was obtained using the readout streak method (Page et al. 2013b), as the nova was too bright for normal aperture photometry, described in Poole et al. (2008). Each image was inspected and those where the spacecraft failed to achieve a lock-on were discarded. The observations were 


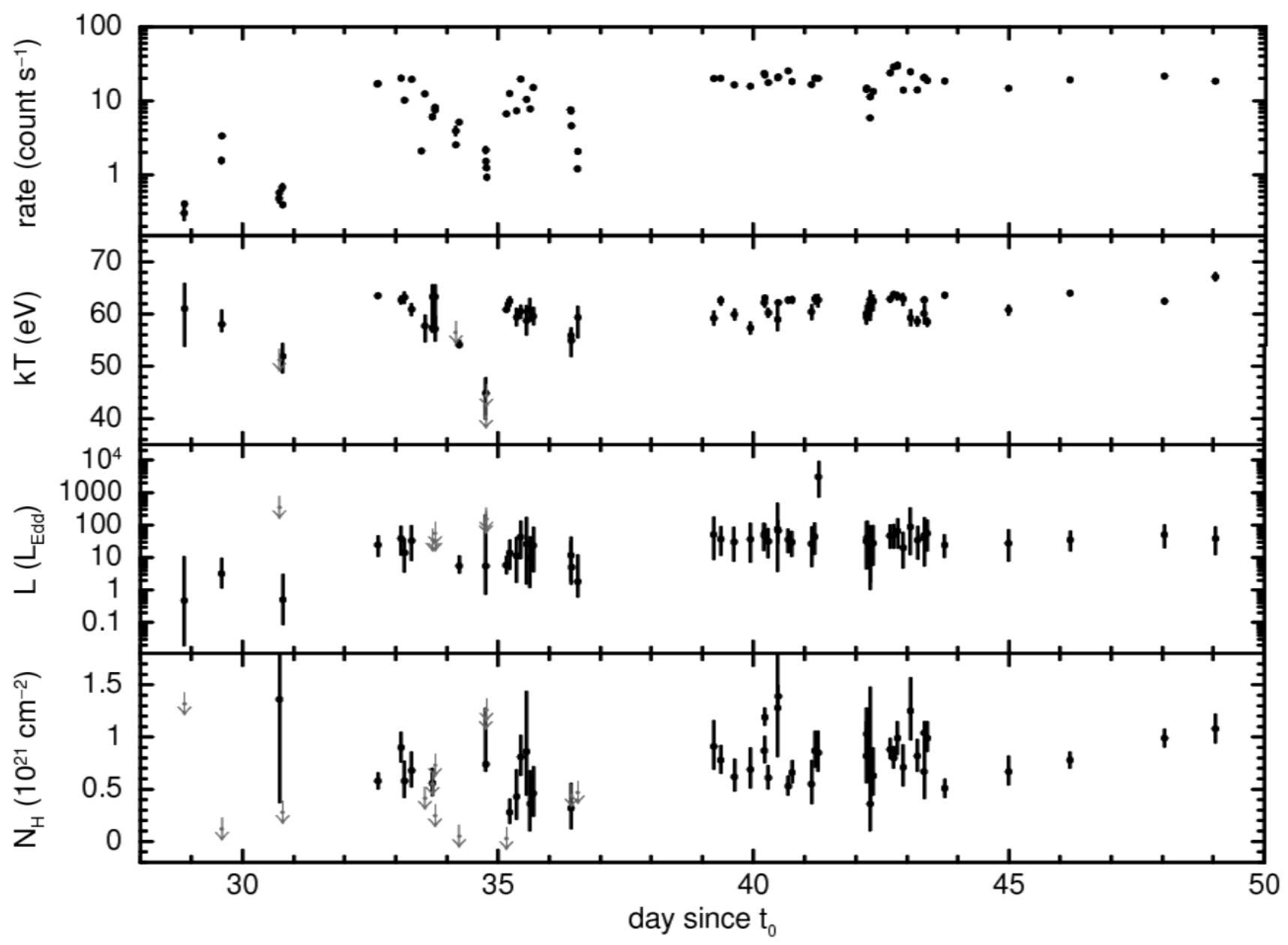

Figure 11. As for Figure 10, but a zoomed-in version for the observations up to day 50 showing the early variability more clearly.

mainly in the uvw2 band, with additional observations in the UVOT uvm2 and uvw1 bands, with central wavelengths: $u v w 2=1928 \AA ; u v m 2=2246 \AA ; u v w 1=2600 \AA$. Figure 12 illustrates the UVOT light-curve. There is a plateau in the UV emission between at least days 90 and 170, approximately coinciding with the main interval of bright, hightemperature, super-soft X-rays (Figure 10. While it is hard to be definitive, due both to data gaps and small scale variability, the plateau in the uvw2 filter may start slightly later and end slightly earlier than the soft X-ray plateau. Such a plateau phase has been seen in other novae (e.g. Kato, Hachisu \& Luna 2008, Page 2013). Note that the SMARTS optical photometry shows a plateau as well, starting about day $\sim 100$ (consistent with uvw1), and ending after day $\sim$ 130, during solar conjunction (see Figure A1.

We looked for temporal structure in the uvw2 photometry by computing the $\mathrm{n}=2,3$, and 4 structure functions using the method described in Saxton et al. (2012). No evidence of a preferred timescale in the range of 0.01-10 d was found.

\section{$5.2 \quad$ Swift UVOT spectroscopy}

The initial Swift UVOT observations, which were obtained on 2016 October 15, 6.1 days after the eruption, used the
UV grism. On the next two days, two further UV spectra were obtained (Kuin et al. 2016). The nova brightness was just below the limit where the flux calibration is possible (Kuin et al. 2015). The initial Swift Target of Opportunity observations were taken with no offset, slew in place, or photometry, to allow for quick scheduling of the observation.

Starting on 2016 October 21, we continued with UV grism clocked-mode (Kuin et al. 2015) observations every two days as scheduling allowed. These were typically taken at an offset of $5.6^{\prime}$ vertically in the detector plane so that the overlap of zeroth orders of field stars is limited to wavelengths shorter than about $2100 \AA$. For the periods 2016 November 7-14 and 2016 December 31 to 2017 January 6, the observations were made at no offset, and during the period of 2016 November 23 to December 12, the offset varied with the roll angle. The main effect is that in the spectra with no offset there are clear signatures of second order emission lines present, namely those at $1750 \AA$, $1909 \AA$, and $2143 \AA$ in second order appear around $2880 \AA$, $3310 \AA$, and $3910 \AA$ in the first order. These emission lines have also been found in other CO novae, like nova V339 Del (Page et al. 2014 Shore et al. 2016). The line at $3130 \AA$ is likely the O III 3133.7 A resonance line (Young, Feldman \& Lobel 2011) and is being fluoresced by the He II 304 EUV line. For that reason it is not affected by optical thickness effects as the other 


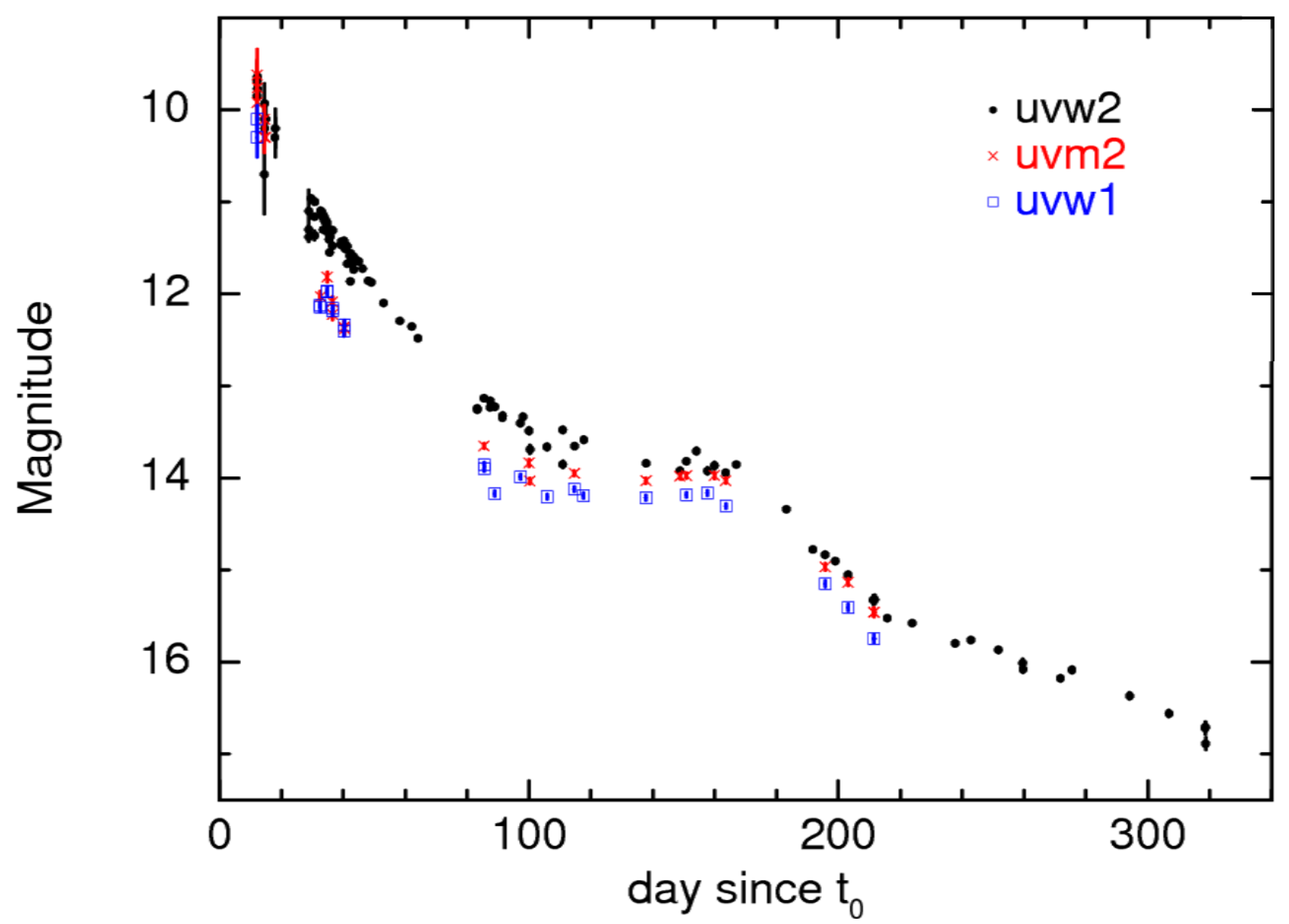

Figure 12. The UVOT light-curve of nova SMCN 2016-10a plotted against time in days since $t_{0}$.

resonance lines are. The O II $2471 \AA$ line is probably present, but weak and blended with other weak features.

The spectra were extracted from each image using the UVOTPY software (Kuin 2014) with the calibration of Kuin et al. (2015). The grism images were inspected to identify contamination from zeroth and first orders of other sources. The spectra were summed in groups of 3 to 5 exposures to improve the continuum which retains a noise contribution from irreducible MOD-8 noise (Kuin et al. 2015). Prior to summing, the individual spectra were lined up by shifting them in pixel/bin space, re-computing the wavelengths after the shift. This is needed as the dispersion changes from the short to long wavelengths. The dates of the summed spectra are provided in Table A7. The spectra are illustrated in Figure 13. Up to day 9 there is unresolved absorption in the 2550-2650 $\AA$ range consistent with the Fe-curtain (optically thick) still being weakly present. The UV spectra show strong emission from N III] $1750 \AA$, C III] $1909 \AA$ A, N II $2143 \AA$, C II $2324 \AA, \mathrm{Mg}$ II $2800 \AA$, and [Ne III] $3868 \AA, 3967 \AA$. Up to day 43 we see the Al III $1860 \AA$ resonance line, while the lines from O III $3130 \AA$ and [Ne V] $3346 \AA, 3426 \AA$ become prominent after day 28, suggesting a gradual increase in ionization. After day 117 post-eruption, the UV grism data (4 ks summed exposure) become too noisy showing only evidence of the continued presence of the N III] $1750 \AA$ and C III] 1909 A lines.
The line flux was determined by integrating the flux above the nearby continuum. Some lines were difficult to measure. The [Ne v] $3346 \AA$ line suffers from the nearby C III] $1909 \AA$ line in the second order which is offset from the first order, but presents such a large coincidence loss pattern that it causes a re-distribution of the line flux (e.g. Kuin et al. 2015). We measured the flux across the pattern. Figure 14 illustrates the evolution of the line fluxes with time.

The Mg II $2800 \AA$ and C III] $1909 \AA$ were compared to establish that no coincidence loss affects the C III] $1909 \AA$ line fluxes. The reason we believe that coincidence loss is corrected appropriately is that the $\mathrm{Mg}$ II $2800 \AA$ line flux is comparable and lies in the much more sensitive range of the grism where it would be affected more.

Having established this, the initial rise in the CIII] $1909 \AA$ flux prior to day 16 is real and different from the flux changes seen in the $\mathrm{Mg}$ II $2800 \AA$ resonance line. Other resonance transitions are present, N II $2143 \AA$, C II $2324 \AA$, and $\mathrm{Al}$ III $1860 \AA$, and they all show a similar behaviour, where the line flux shows a decrease from the first spectrum observed at day 6 .

In the O III $3130 \AA$ line, which is probably optically thin because it is caused by fluorescence from the He II 304 EUV line, we observe a nearly straight slope, whereas in the permitted lines the flux decrease is initially steeper to join in the same rate of decrease as all lines some time after day 20. 


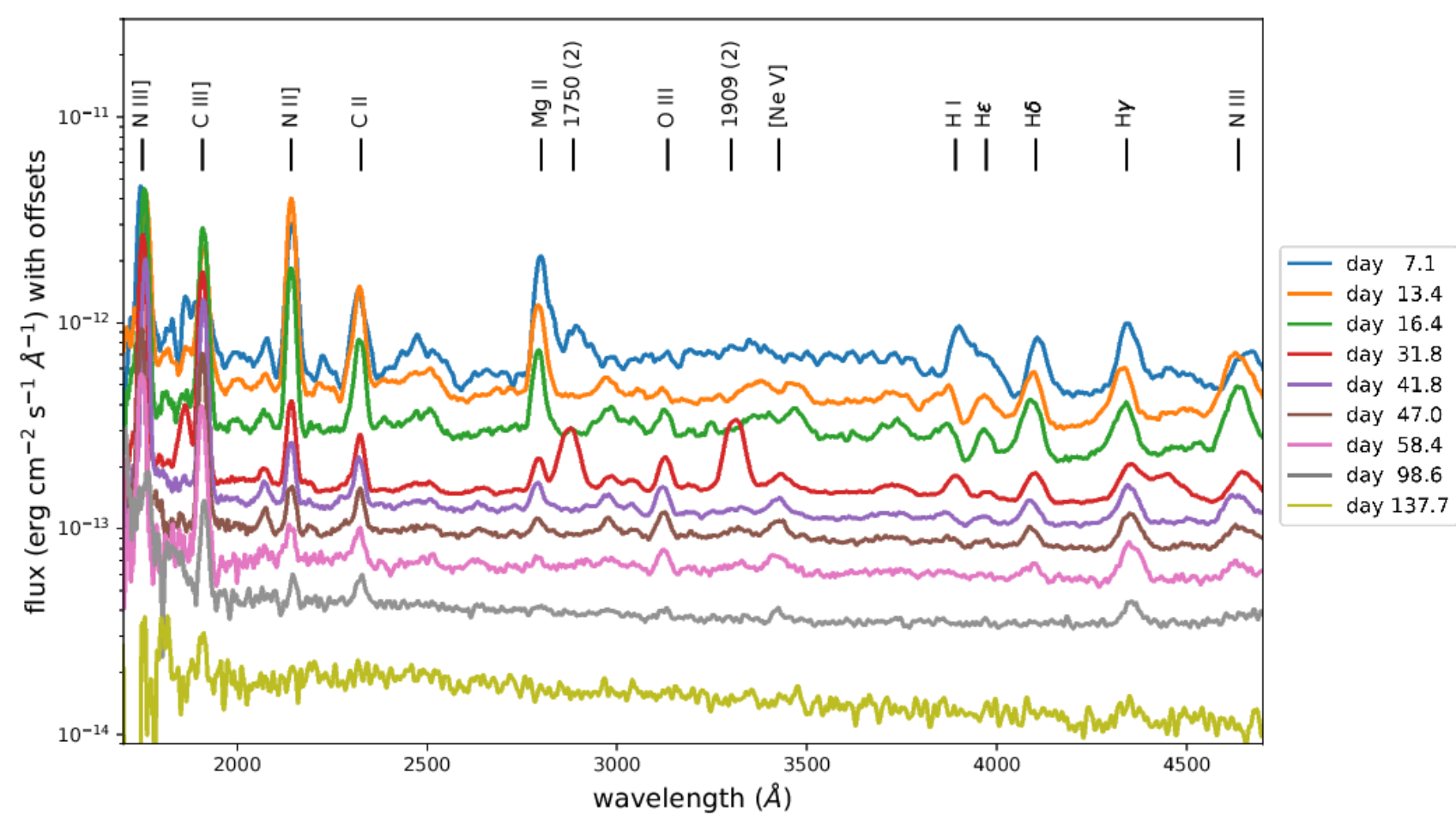

Figure 13. The UV grism summed spectra. For clarity the spectra are all shifted vertically, starting with an offset of $1 \mathrm{e}-14$ then incrementally by $2 \mathrm{e}-14$ in flux. The numbers to the right are days since eruption $\left(t_{0}\right)$. A colour version of this plot is present in the online journal.

As in the photometric data, after day 60 the slope changes, and the line flux evolution is seen to be consistent with the photometric light-curve (see Figure 12) taking into consideration the large uncertainties due to the large $\mathrm{S} / \mathrm{N}$ in the late time spectra.

The different temporal development of the line fluxes is likely due to opacity. In the resonance lines we can approximate the emission originating from an optical depth of one. Comparison of the temporal development of the optically thick lines to the optically thin line can be interpreted that the initial flux in the optically thick lines is higher. This can be explained if the flux source of the optically thick lines is dependent on the earlier and higher flux. Assuming that the ejecta are moving at a nearly constant speed, so that there are no frequency shifts, the optically thick line may 'bottle-up' some of the earlier high flux radiation. The most likely mechanism is back-scatter of the WD radiation by the ejecta. By that means the radiation density in the line is enhanced within the confines of the ejecta and the flux in the line is partly from the earlier radiation density. When the line becomes effectively optically thin (i.e., photons will escape through the shell, even when the optical depth is still larger than one), the temporal development of the line flux in the optically thick lines will start to match the optically thin line.

What this tells us is that the ejecta up to at least day 20 are not transparent, consistent with the SSS flux not being observed until day 28 . It also suggests that the central source is still mostly covered by optically thick ejecta in all directions in these lines, i.e, there is no large hole in the ejecta letting the back-scattered radiation field escape before that time. In the context of asymmetric ejecta (Shore et al. 2011), this means that at least some matter is ejected in all directions, though the mass in certain directions may be much larger.

\section{DISCUSSION}

\subsection{Distance Modulus}

While the mean distance to the SMC is around $61 \mathrm{kpc}$ this galaxy is very extended in the line-of-sight (Caldwell \& Coulson 1986 Jacyszyn-Dobrzeniecka et al. 2016), with Cepheid variables distributed from $<54 \mathrm{kpc}$ to $>76 \mathrm{kpc}$. In this Section we will try to derive an estimate of the distance to the nova using the available methods and test at the same time the usefulness of these methods.

Buscombe \& de Vaucouleurs (1955) suggested that all novae have a similar absolute magnitude 15 days after eruption. Cohen (1985) estimated this as $M_{V, 15}=-5.60 \pm 0.45$ using 11 objects, while Downes \& Duerbeck (2000) refined the value to $M_{V, 15}=-6.05 \pm 0.44$ using 28 objects. Interstellar reddening towards the SMC is small and for our analysis we assume $A_{V}=0.11 \pm 0.06$ and $A_{I}=0.07 \pm 0.06$, using relations (4) and (5) in Haschke, Grebel \& Duffau (2012) and the average value of $E(V-I)$ for the SMC from Haschke, Grebel \& Duffau (2011). Using the EWs of the Na I D absorption lines (Figure $15-\mathrm{EW}_{\mathrm{D} 1}=0.13 \pm 0.02$ and $\mathrm{EW}_{\mathrm{D} 2}=$ $0.17 \pm 0.02)$, and their relationship to the interstellar extinction (as specified by Munari \& Zwitter 1997 and Poznanski, Prochaska \& Bloom 2012) we obtain values of $A_{V}$ consistent 


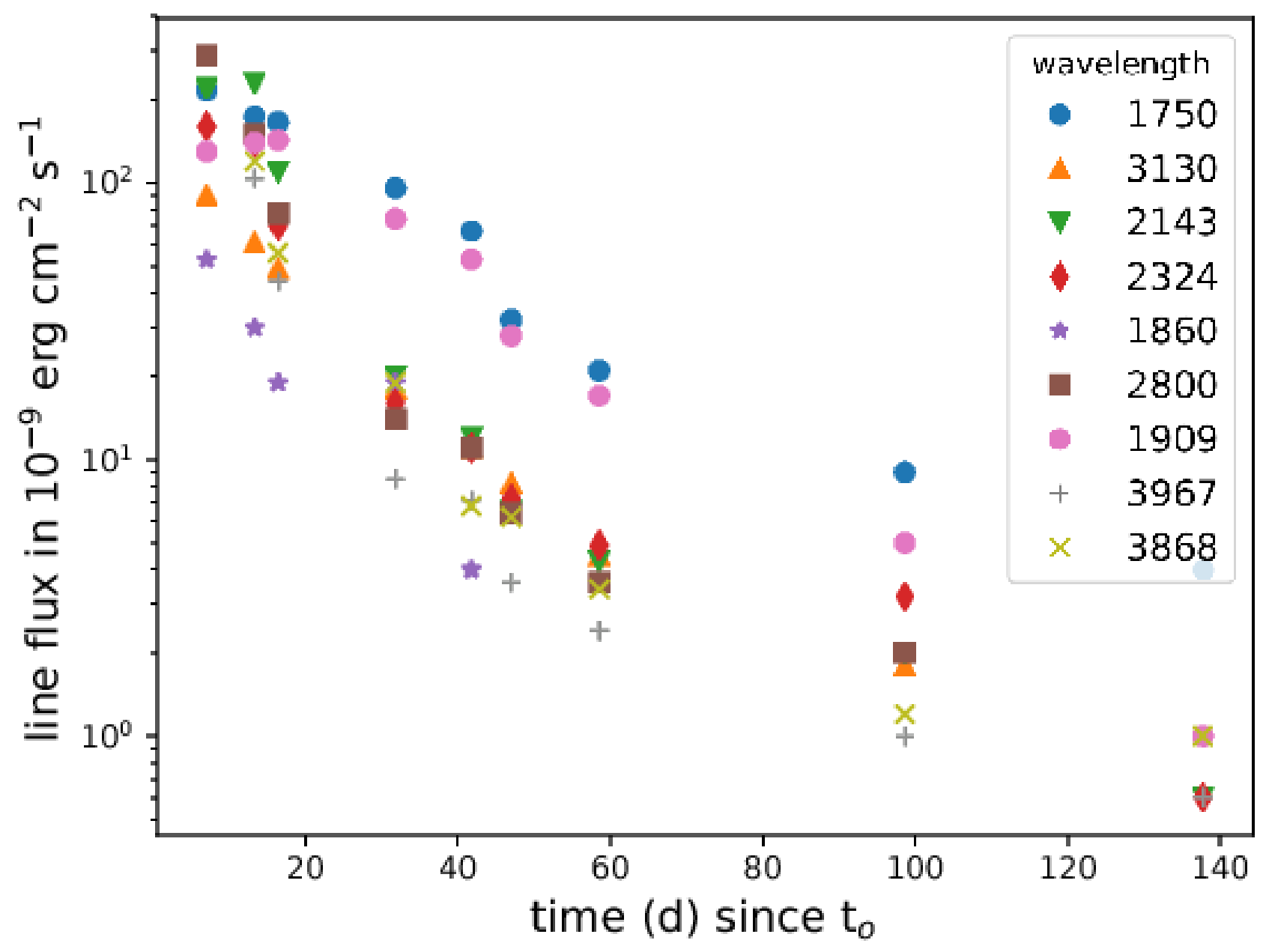

Figure 14. The evolution with time post-eruption of the line fluxes in the UVOT spectra, colour and symbol coded based on the lines rest-wavelength.

with our assumptions. Most likely the reddening contribution from the SMC is very low (see Section 6.3.1 and the discussion about the radial velocities of the Na I D absorption lines).

At day 15 from the eruption this SMC nova was at an apparent magnitude $V=12.30 \pm 0.01$ or $V_{0}=V-A_{V}=$ $12.19 \pm 0.07$. Using the Downes \& Duerbeck (2000) relation this gives $(m-M)_{0}=18.23 \pm 0.51$ and a distance $d \sim$ $44_{-9}^{+11} \mathrm{kpc}$.

After a survey of 24 novae in M87, Shara et al. (2017b) recently found that the minimum scatter of novae absolute magnitude occurs at day 17 in the $V$-band and day 20 in the $I$-band. Using the Hubble Space Telescope F606W and $\mathrm{F} 814 \mathrm{~W}$ filters for the $V$ and $I$ bands respectively, they found $M_{V, 17}=-6.06 \pm 0.23$ and $M_{I, 20}=-6.11 \pm 0.34$. At day 17 from maximum light, nova SMCN 2016-10a was at $V=12.60 \pm 0.01$ or $V_{0}=12.49 \pm 0.07$. Using the absolute magnitude at day 17 from Shara et al. (2017b) gives $(m-M)_{0}=18.55 \pm 0.30$ and a distance $d \sim 51_{-6}^{+7} \mathrm{kpc}$. At day 20 from maximum light, nova SMCN 2016-10a was at $I=12.08 \pm 0.01$ or $I_{0}=I-A_{I}=12.01 \pm 0.07$. Using the absolute magnitude at day 20 from Shara et al. (2017b) gives $(m-M)_{0}=18.12 \pm 0.41$ and a distance $d \sim 42_{-7}^{+8} \mathrm{kpc}$.
The nova is located about two degrees south of the central body of the SMC and outside of the region examined by Subramanian et al. (2017). Nevertheless, it is worth noting that these authors identify a red clump population at $\sim 12 \pm 2 \mathrm{kpc}$ in front of the main body of the SMC, that may have been the result of tidal stripping. Other explanations for the discrepancies in the distance: (1) the validity of using $M_{V, 15}$ as a distance indicator which was questioned by several authors (see e.g. Jacoby et al. 1992 Ferrarese, Côté \& Jordán 2003; Darnley et al.|2006); (2) These relations are supposed to give a rough estimate of the distance and not accurate values; (3) underestimating the absolute magnitude derived using the relations of Downes \& Duerbeck (2000) and Shara et al. (2017b). The latter derived their relations using a sample of novae in M87, hence, a parent galaxy with different metallicity than the SMC; (4) Although, the extinction is not expected to play a major role relative to the direction of the nova, an overestimation in the extinction values adopted can lead to a slight underestimation in the distance $(\sim 2 \mathrm{kpc})$. We conclude that the nova is within the SMC and possibly foreground of the bulk of the stars in the field. A less likely possibility that the nova belongs to the Galaxy is discussed in Section 6.3.1. On the 


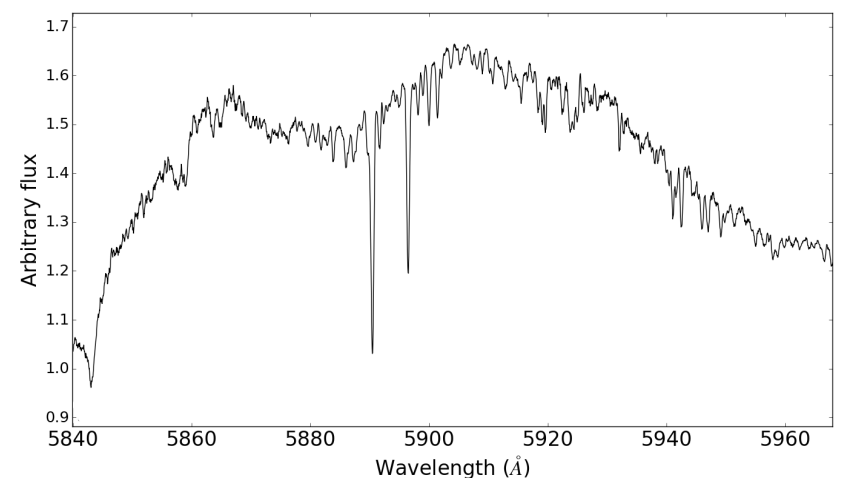

Figure 15. The NaI D doublet absorption lines D1 (5896.6 ̊) and D2 (5890.6 $)$ from the high resolution spectrum of day 8 .

Finding charts (North is up and East is to the left, image size $2^{\prime} \times 2^{\prime}$ )
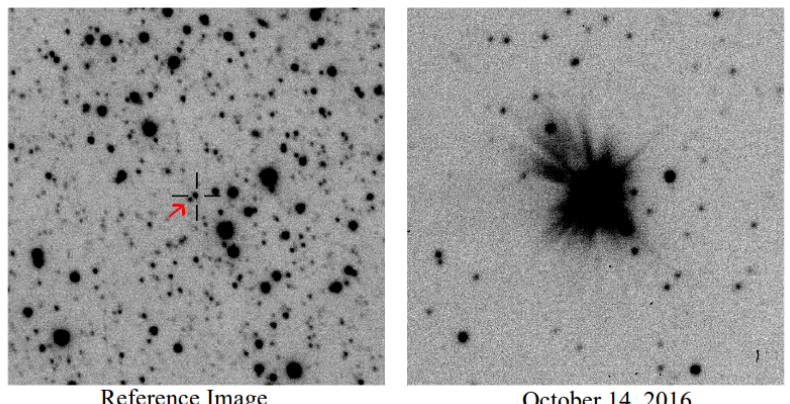

Figure 16. Left: OGLE finding chart of the progenitor system before the eruption. Right: image of the field 5 days after the eruption.

assumption that it is associated with the SMC we will adopt a distance of $61 \pm 10 \mathrm{kpc}$ throughout the rest of the paper. This distance covers the extended SMC and the distances we derived under uncertainties.

\subsection{The progenitor system}

During quiescence, the emission from a CN system is mainly from the secondary star and the accretion disk (depending on its inclination relative to the line of sight). The emission from the WD is almost negligible during quiescence. The OGLE survey has been monitoring the target since 2010. Figure 16 shows a finding chart of the object during quiescence (left) and an image of the object during the eruption (right). The star South-East of the nova (indicated with the red arrow) is $\sim 2.1$ arcsec away.

In quiescence, the target shows irregular variability that might be due to variations in the mass-transfer rate (see Figure 11. No periodic variability appears in the light-curve of the progenitor that can lead us to constrain the orbital period of the system. This means that the orbital plane of the system may be oriented face-on to the observer, so that the contribution of the disk to the total emission from the system might be large. During quiescence, the progenitor is observed at $\langle V\rangle \sim 20.70$ and $\langle I\rangle \sim 20.55$. In Figure 17 we present the variation of $V-I$ over time during quiescence. We only include the days when the magnitudes of both

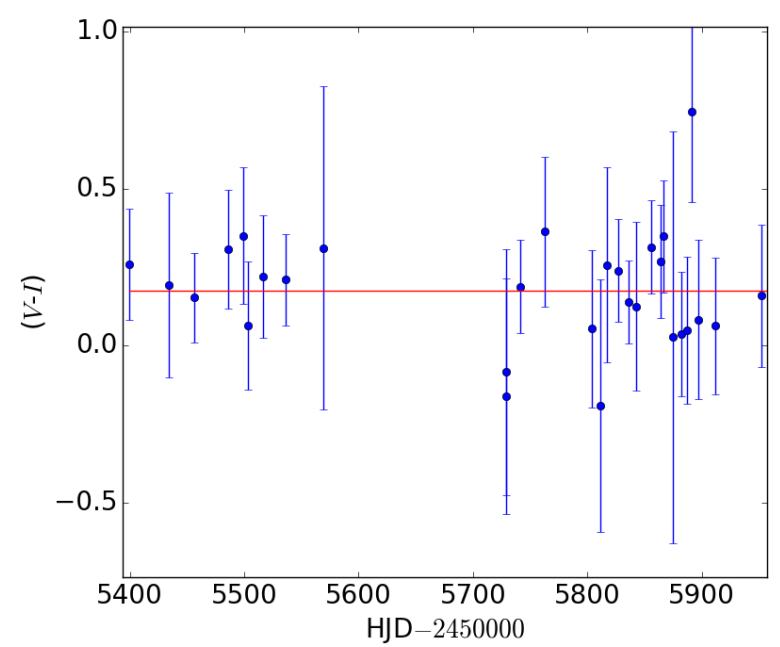

Figure 17. The evolution of $(V-I)$ during quiescence. The red line presents the mean values of $(V-I)$.

broadband colours are measured quasi-simultaneously. The $V-I$ colours are more sensitive to the disk than to the secondary, if the latter is a main sequence or sub-giant. However, these colours would be equally sensitive to a red giant secondary. The changes in $V$ and $I$ during quiescence (Figure 1) and the moderately high values of $V-I$ might reflect the contribution of a bright disk that is showing temporal variability.

We found several sources less than $\sim 1.0^{\prime \prime}$ from the nova in archival data from: USNO-B1 catalogue (Monet et al. 2003) ( $B=20.17$ and $R=20.0)$; the Magellanic Cloud Photometric Survey (MCPS; Zaritsky et al. 2002) $(U=19.71 \pm 0.14, B=20.44 \pm 0.07$, and $V=$ $20.58 \pm 0.04$ ); GALEX catalogues (Bianchi et al. 2011) (NUV $=20.57 \pm 0.20$ and FUV $=20.53 \pm 0.33$ ). After checking several charts and images of the field from OGLE, MASTER and Simbad (Wenger et al. 2000), it seems likely that all these observations refer to the nova progenitor. These data along with the OGLE data, allow us to place the progenitor on $B$ versus $B-R, V$ versus $B-V, I$ versus $B-I$ and $I$ versus $V-I$ colour-magnitude diagrams (CMDs, Figure 18. The $B$ versus $B-R$ indicates a system which may have a sub-giant companion (SG-novae). However, the three other CMDs indicate a system with a main sequence companion (MS-novae). The inconsistency of the $B$ versus $B-R$ with the other plots could be understood if the progenitor had strong $\mathrm{H} \alpha$ emission. It is worth noting that $B, V, R$, and $I$ bands are very sensitive to the emission from the disk. The optical colours depend sensitively on the ratio of the disk to stellar photospheric emission.

Darnley et al. (2012) show that, ideally, placing the system on a CMD using NIR colours can give us an insight into the secondary star type. The NIR colours are more sensitive to the emission from the secondary than that from the disk. With lack of observations in NIR for the progenitor in the archival data, it is difficult to say much about the secondary star. 

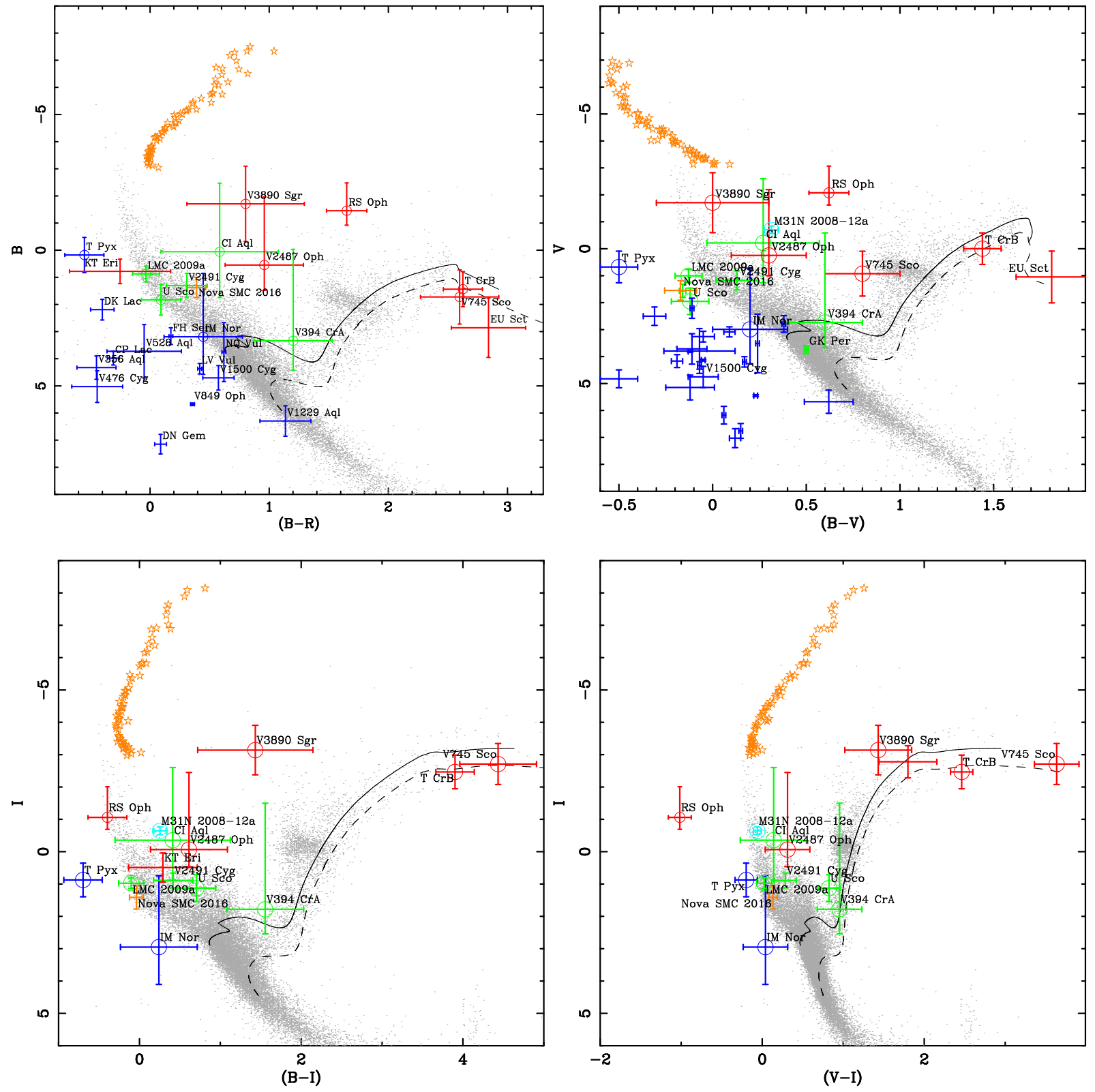

Figure 18. Colour-magnitude diagrams showing stars (grey dots) from Hipparcos data set (Perryman 1997) with parallax errors < $10 \%$. These stars have been transformed to the distance and extinction of the SMC. BVI photometry is taken directly from the Hipparcos catalogue, $R$ photometry is taken from the USNO-B1 catalogue (Monet et al. 2003 via the VizieR database, Ochsenbein, Bauer \& Marcout 2000). The blue points represent Galactic MS-novae, the green points represent Galactic SG-novae and red points represent Galactic red giant novae (RG-novae) (see Schaefer 2010, Darnley et al. 2012, and references therein). The known recurrent novae in this sample have been identified with an additional circle. Orange points represent nova SMCN 2016-10a during quiescence. The orange stars indicates the track of nova SMCN 2016-10a from day 11 till day 108 post-eruption. M31N 2008-12a is shown in light blue due to its uncertain donor. A colour version of this plot is present in the online journal.

\subsection{Eruption and early decline}

The system was last observed by OGLE before the eruption on 2457667.67 at $V=20.65 \pm 0.03$. Adopting a distance of $61 \pm 10 \mathrm{kpc}$ and $A_{V}=0.11 \pm 0.06$ will result in $M_{V}=+1.61 \pm 0.45$ three days before the eruption. Although the MASTER VWF camera measurements are not precise $V$-band photometry, the measurements can be considered as an approximation of $V$-band photometry (see Section 2.3. Hence, we consider $V_{\max } \approx 8.5 \pm 0.1$ which results in $M_{V, \max } \approx-10.5 \pm 0.5$. We can thus constrain an approximation for the eruption amplitude as $A \approx 12.1 \pm 1.0$. The amplitude of the eruption is not only related to the eruption itself, but also to the luminosity of the donor during quiescence. Hence, such a high eruption amplitude might also indicate a sub-luminous donor, such as a main sequence one. The eruption amplitude agrees well with the amplitudes given in the $A$ versus $t_{2}$ relationship for CNe in Warner (1995b), where CNe of similar speed class to SMCN 


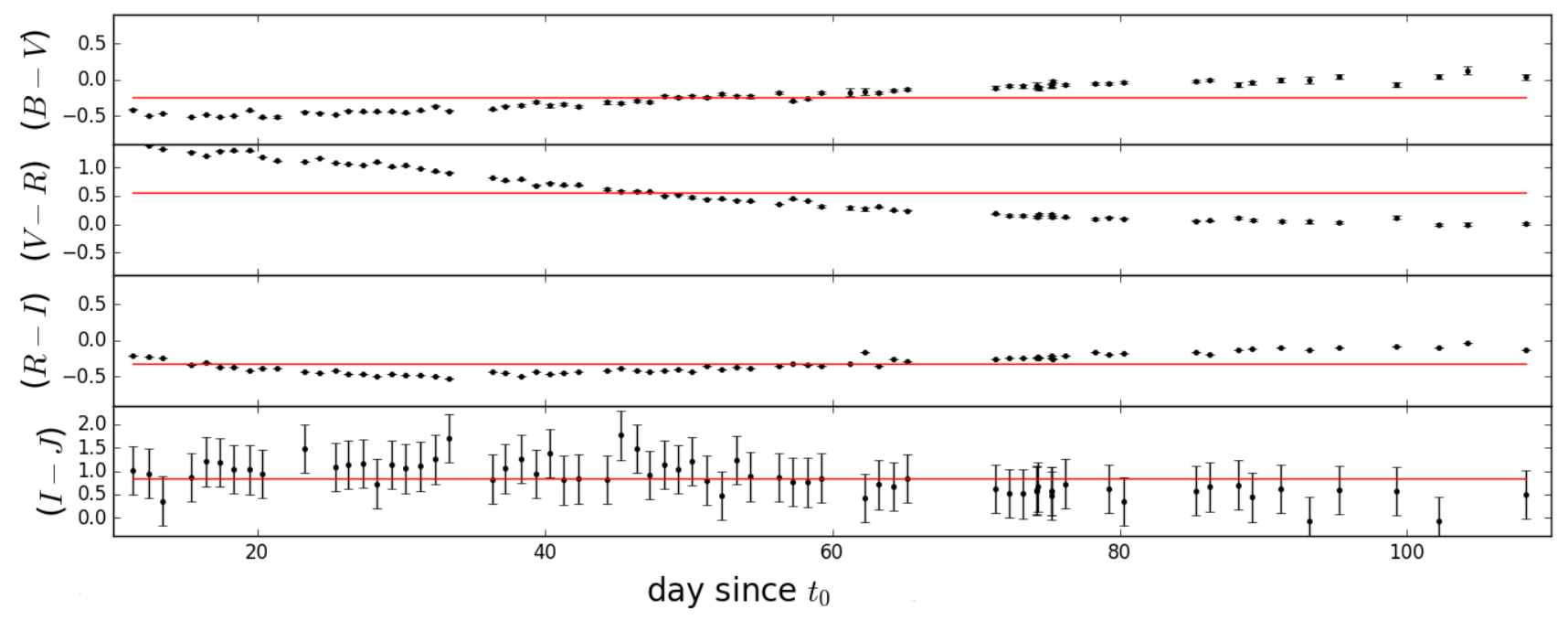

Figure 19. Optical and NIR broadband colour evolution of nova SMCN 2016-10a between day $\sim 11$ and day $\sim 100$ post-eruption. The red line is the average of the values between the two dates.

2016-10a, with a main sequence donor, are characterized by $A \geqslant 12$.

\subsubsection{The brightest nova discovered in the SMC?}

If nova SMCN 2016-10a is indeed in the SMC, $M_{V, \max } \approx-10.5 \pm 0.5$ will make it the brightest nova discovered in the SMC, and probably one of the brightest ever discovered. At $M_{V, \max } \approx-10.5 \pm 0.5$ it is at least as bright as V1500 Cyg and CP Pup. These two novae are considered the brightest nova events ever recorded (see e.g. Warner 1985 Stockman, Schmidt \& Lamb 1988; Hachisu \& Kato 2006 Shafter et al. 2009). If the nova is in the foreground of the SMC at $\sim 46 \pm 10 \mathrm{kpc}$ (the mean value of the three distances derived in Section 6.1), we derive $M_{V \max } \approx-9.9 \pm 0.6$ meaning it is still a very luminous nova $\left(M_{V \max } \leqslant-9.0\right.$; Shafter et al. 2009). If the nova belongs to our Galaxy, which is very much less likely, then $M_{V \max }$ is greater than -6.0. In this case the nova may be under-luminous and possibly classified as a faint fast nova (see e.g. Kasliwal et al. 2011, Shara et al. 2017a).

The first value is in good agreement with the SMARTS $V$-band photometry. The nova magnitude in the $V$-band has been measured as $12.14\left(V_{0}=12.03 \pm 0.06\right)$ at $t=11.3 \mathrm{~d}$. We estimate $t_{3}=7.8 \pm 2.0 \mathrm{~d}$ which is $<11.3 \mathrm{~d}$, meaning that the nova reached a maximum brightness in the $V$-band smaller than $12.03 \pm 0.06-3.0=9.03 \pm 0.06$. This value leads us to constrain a maximum on $M_{V, \max }<-9.9 \pm 0.5$ (at $d=61 \pm 10 \mathrm{kpc}$ ).

In order to constrain the distance to the nova, we derived radial velocities from the $\mathrm{NaI} D$ doublet absorption lines at $5890 \AA$ and $5896 \AA$. The lines are detected at $\sim 5890.6 \AA$ and $\sim 5896.6 \AA$, showing a red-shift of $\sim 16 \pm 2 \mathrm{~km} \mathrm{~s}^{-1}$ in all five HRS spectra. The origin of these lines is either circumstellar or interstellar material. The line widths are too narrow to be produced in an outflow and hence they come from a static region (see Figure 15). Since the average radial velocity of the SMC is $\sim 160 \mathrm{~km} \mathrm{~s}^{-1}$ (Neu- gent et al. 2010), these absorption lines are due to Galactic interstellar medium absorption. Note that if these absorption lines originate from the SMC, they should be at $\sim$ $5893 \AA$ and $\sim 5899 \AA$. In conclusion, none of the three possibilities of the distance to the nova can be ruled out. But it is still very likely that the nova is in the SMC or foreground of the SMC, and the interstellar absorption is from the Milky Way.

\subsubsection{Post-eruption colour evolution}

In Figure 19 we present the evolution of different broadband colours during the early decline until $\sim 100$ days post-eruption. We only include the days when the magnitudes of different broadband colours are measured quasisimultaneously.

For objects that show emission lines in their spectra, the emission lines can be a significant contributor to the flux. Hence, the evolution of the colours is an indication of the evolution of both the lines and the continuum (Bode et al. 2016). In the optical, the emission lines contribute strongly to the $B$-band flux, especially the Balmer lines $(\mathrm{H} \beta, \mathrm{H} \gamma$, $\mathrm{H} \delta$, and $\mathrm{H} \epsilon$ ) during the early days after the eruption. In the $R$-band, $\mathrm{H} \alpha$ is a strong contributor to the flux. The O I lines are also an important contributor to the $I$-band flux during the early decline. The $B-V$ broadband colour evolution starts bluer at $\sim 11$ days after eruption, showing a monotonic evolution toward redder colours until 100 days post-eruption. This evolution of $B-V$ can be explained by the dimming of the Balmer emission lines during the decline from maximum light which affect the flux in the $B$-band, followed by the emergence of the nebular [O III] lines, at the blue edge of the $V$ band, after the start of the nebular phase (see section 6.5.

The evolution of $V-R$ starts redder and becomes bluer monotonically with time, which in turn can also be attributed to the dimming of $\mathrm{H} \alpha$. The $R-I$ evolution shows slight variation around the mean, probably due to the rela- 


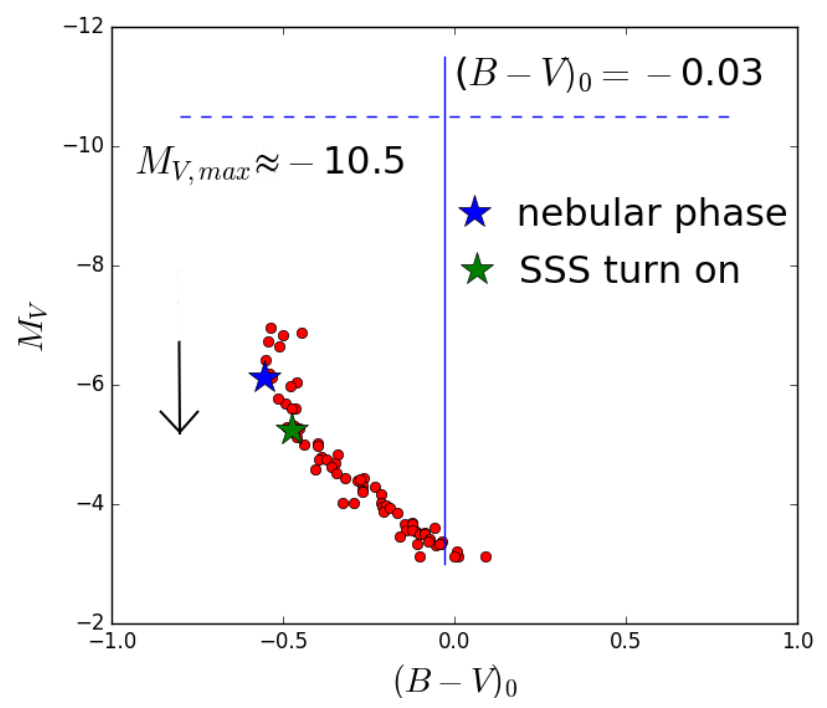

Figure 20. Colour-magnitude diagram of SMCN 2016-10a showing $M_{V}$ against $(B-V)_{0}($ red dots $)$ at a distances $d=61 \mathrm{kpc}$. The evolution spans from day 12 to day $\sim 100$ post-eruption. The black arrow indicates the evolution of $M_{V}$ with time. The blue horizontal dashed line indicates the maximum absolute magnitude. The blue vertical line indicates the intrinsic colours for an optically thick wind free-free emission (see Hachisu \& Kato 2014 and Darnley et al. 2016 for further details). The green and blue stars represent the start of the SSS phase (day 28) and the nebular phase (day 20) respectively. A colour version of this plot is present in the online journal.

tive variation of the $\mathrm{O}$ I lines compared to $\mathrm{H} \alpha$. In the NIR, the evolution of the broadband colours show no trend with time. They show slight random changes around the mean, but the uncertainty on the data is very high and hence it is difficult to say anything about the variation of these colours.

Hachisu \& Kato (2016b) have demonstrated that the evolution of the colours, during and after the eruption, is different for systems hosting a red giant companion (RG-novae) than for SG-novae or MS-novae. They showed that for the RG-novae, the colour evolution follows a vertical track, on the CMD, along the two lines of optically thick wind and optically thin wind free-free emission intrinsic colours, respectively $(B-V)_{0}=-0.03$ and $(B-V)_{0}=0.13$ (see also Hachisu \& Kato 2014, Darnley et al. 2016). For SG- and MS-novae, the colour-magnitude tracks show an evolution towards bluer colour to reach a turning point, close to the start of the nebular phase, from where the colours evolve backward and become redder.

In Figure 20 we present a CMD, illustrating the evolution of $M_{V}$ against $(B-V)_{0}$, for nova SMCN 2016-10a. The track shows an evolution similar to novae with a sub-giant or a main sequence secondary (see figure 7 in Darnley et al. 2016). Although we lack broadband observations during the first 10 days after maximum optical light, the available data show a blue-ward evolution before the turning point into a red-ward evolution near the start of the nebular phase. The evolution of the CMD can be interpreted as follows: at the very early phase after maximum optical peak, the colours show a gradual change towards the blue, corresponding to the free-free emission phase of the nova spectrum. Later, after maximum, the colours becomes bluer due to the strong emission lines within the $B$-band. Then, these lines start to fade and the turning point starts. Close to the start of the nebular phase, the colours turn red-ward due to the emergence of nebular lines such as [O III] $4959 \AA$ and $5007 \AA$ (see Section 6.5) at the blue edge of the $V$-band (Hachisu \& Kato 2016b).

Figure 21 illustrates distance- and extinction- corrected spectral energy distribution (SED) plots, showing the evolution of the post-eruption SED of nova SMCN-2016-10a. SMARTS and Swift UVOT data are combined to form the SED across the NIR, optical, and UV spectral range. We only plot the data that have corresponding observations in all the bands. In the optical, the evolution of the SED plots is affected by the line emission as discussed for the CMD and colour evolution plots. $\mathrm{H} \alpha$ contributes strongly in the, initially bright, $R$ band. Since nova behaviour during and after peak is similar to blackbody emission (see e.g. Gallagher \& Ney 1976, Bode \& Evans 2008), we can conclude from Figure 21 that the peak of emission is beyond the uvw2 of Swift UVOT (shorter than $2000 \AA$ ) throughout the 12-100 day interval. At optical maximum the spectra of CNe show a blackbody emission peaking in the optical with temperatures around 10,000 K (see e.g. Bode \& Evans 2008 and references therein). A simplified model of an evolving pseudo-photosphere, in the optically thick ejecta, has been adopted to re-produce the evolution of the post-eruption emission from novae (see e.g. Bath \& Harkness 1989). The temperature of the photosphere reaches its lowest at the optical peak, and then increases with time while the radius of the photosphere shrinks. This increase in the temperature shifts the blackbody temperature towards the UV. Since nova SMCN 2016-10a is a very fast nova and since the first SED plot is around 12 days post-optical-maximum, it is expected that the SED peaks toward the UV. This peak is beyond the uvw2 of the Swift UVOT, which indicates temperatures above $15,000 \mathrm{~K}$.

\subsection{Mass of the WD and the ejected envelope}

It is now well understood that several parameters other than the WD mass affect nova eruptions, including the accretion rate on the WD surface, the WD temperature (and thus luminosity), the WD chemical composition, and the chemical composition of the accreted matter (see e.g. Yaron et al. 2005. Hillman et al. 2014; Shara et al. 2017a, and references therein). However, it is widely accepted that the WD mass is the critical factor in nova eruptions, and it has a great effect on the light-curve morphology and decline rate. Novae with high mass WDs $\left(M_{\mathrm{WD}}>1.0 \mathrm{M}_{\odot}\right)$ are expected to show fast declines in the optical light, while novae with low mass WDs are expected to show slow optical decline rates. The Hillman et al. (2016) models predicted that any novae with $t_{2}<10$ days must contain a WD with a mass $\geqslant 1.25$ $\mathrm{M}_{\odot}$.

The mass of the accreted envelope is also related to the decline rate and the WD mass. We use the empirical relation from Shara et al. (2017a) to estimate the accreted envelope mass:

$$
\log M_{\mathrm{env}}=0.825 \log \left(t_{2}\right)-6.18,
$$

resulting in $M_{\text {env }}=(0.20 \pm 0.05) \times 10^{-5} \mathrm{M}_{\odot}$, which is in good 


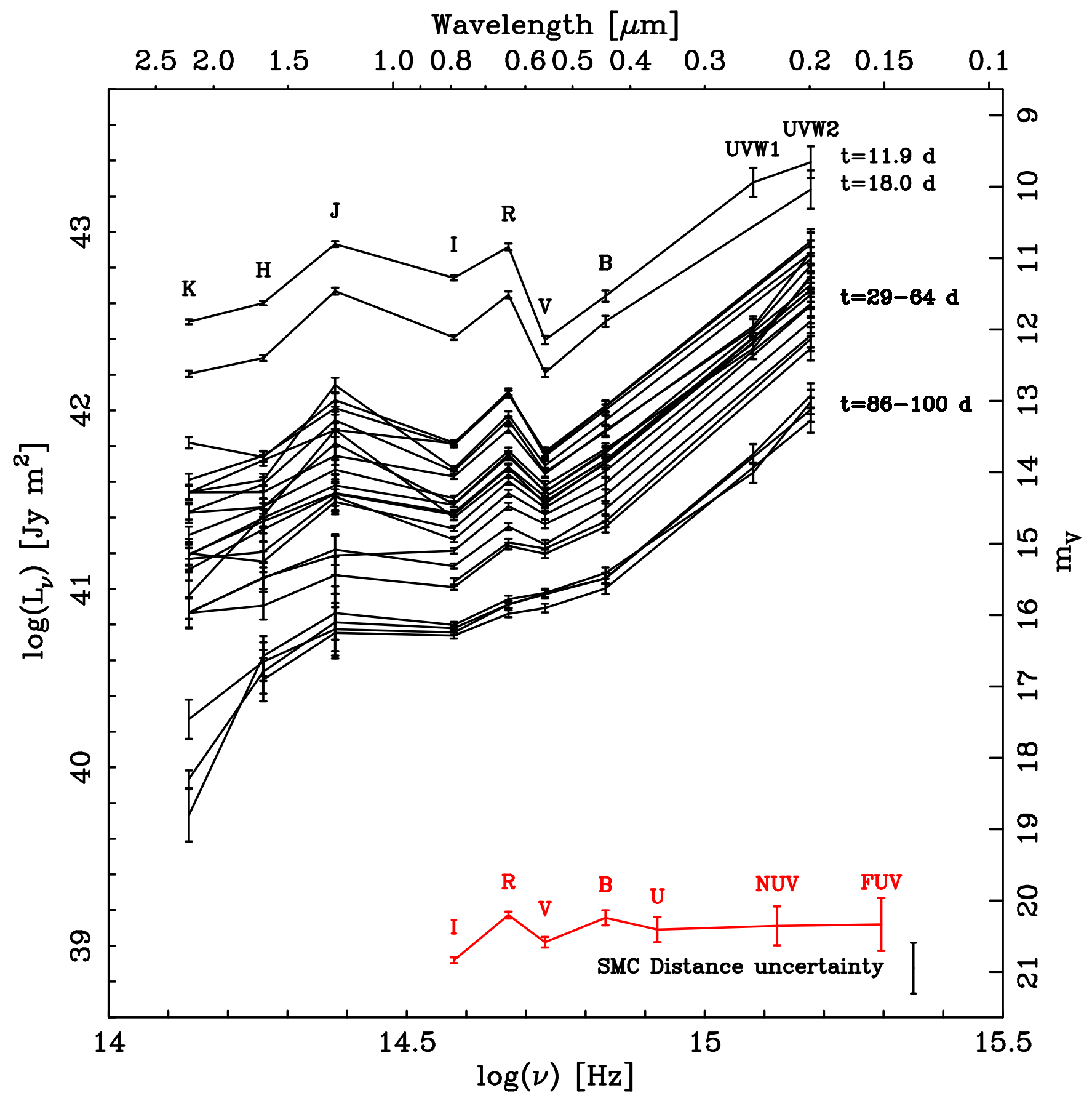

Figure 21. Distance- and extinction-corrected SED plots showing the evolution of the SED of nova SMCN 2016-10a eruption. The error bars include contributions from the photometric and distance uncertainties. Red data at the bottom present the quiescence SED, using the MCPS $U B V$, USNO-B1 $R$, OGLE $I$ and GALEX NUV/FUV.

agreement with Hachisu \& Kato (2016b) models for fast novae. One should note that the empirical relation presented in Shara et al. (2017a) was derived by fitting novae from M87, M31, LMC, and the Milky Way.

\subsubsection{SSS and $M_{\mathrm{WD}}$}

The timescale of the SSS phase and uncovering the X-ray source is proportional to the hydrogen ejected envelope mass and inversely proportional to the ejection velocity. Krautter et al. (1996) relate the SSS phase turn-on to the mass of the ejected $\mathrm{H}$ envelope and the ejecta velocity by: $t_{\text {on }} \propto$ $M_{\mathrm{H}}^{1 / 2} v_{\mathrm{ej}}^{-1}$. With relatively low envelope mass and high ejecta velocity, the time since eruption to observe the SSS phase should be relatively short, which is the case for nova SMCN 2016-10a with $t_{\text {on }}=28$ days. This value is comparable to other novae of the same speed class (see e.g. Bode et al. 2016 and references therein). $t_{\mathrm{on}}$ is dependent on the mass of the $\mathrm{WD}$, hence for systems with high $\mathrm{M}_{\mathrm{WD}}, t_{\mathrm{on}}$ is expected to be shorter than for systems with low $M_{W D}$. The SSS phase 
of RS Oph (Osborne et al. 2011), the first nova monitored in detail by Swift, was first detected on day 26 after eruption, very similar to nova SMCN 2016-10a, while V745 Sco (Page et al. 2015) provided an extreme example, where $t_{o n}$ was only $4 \mathrm{~d}$. An extra-galactic perspective is given by nova LMC 2012 (Schwarz et al. 2015), with $\mathrm{t}_{\text {on }}=13 \pm 5 \mathrm{~d}$. All of these novae are likely examples of high WD mass systems. In comparison, nova SMC 2012 (OGLE-2012-NOVA-002) was a much slower system, not seen as a supersoft source until around nine months after the first optical detection (Page et al. 2013a).

The turn-off time, since eruption, for nuclear burning $\left(t_{\text {off }}\right)$ is also strongly dependent on $\mathrm{M}_{\mathrm{WD}}$, more precisely it is inversely proportional to the WD mass. MacDonald (1996) found $t_{\text {off }} \propto \mathrm{M}_{\mathrm{WD}}^{-6.3}$. Novae with similar $t_{\text {off }}$ to that of the nova SMCN 2016-10a are expected to have a WD mass between $1.1 \mathrm{M}_{\odot}$ and $1.3 \mathrm{M}_{\odot}$ (see e.g. Krautter et al. 1996 . Balman, Krautter \& Ögelman 1998, Osborne et al. 2011 and references therein). Despite RS Oph having a similar $\mathrm{t}_{\mathrm{on}}$ to SMCN 2016-10a, its $\mathrm{t}_{\mathrm{off}}$ is noticeably shorter, at $\sim 58$ d (Osborne et al. 2011), implying the WD in the RS Oph system is more massive (see also Brandi et al. 2009, Booth, Mohamed \& Podsiadlowski 2016).

Henze et al. (2014) monitored novae in M31 presenting a series of correlations relating SSS parameters. The values of $t_{\text {on }}=28 \mathrm{~d}$ and $t_{\text {off }}=180 \mathrm{~d}$ lie within the scatter of their results. The luminosity-temperature models in Wolf et al. (2013) (see also Sala \& Hernanz 2005) suggest a high $\left(\sim 1.25-1.3 \mathrm{M}_{\odot}\right)$ WD mass for the $\sim 90 \mathrm{eV}$ SSS temperatures measured for nova SMCN 2016-10a. Thus, the X-ray data agree with the conclusion from the optical that the WD is likely of high mass.

\subsection{Spectroscopic classification}

The spectroscopic and photometric data leave no doubt that the object is a CN eruption. The spectral evolution of nova SMCN 2016-10a shows initially a He/N spectrum dominated by broad prominent Balmer, $\mathrm{He}, \mathrm{N}$, and $\mathrm{O}$ emission lines (Williams \& Darnley 2016). A FWHM of $\sim 3500 \mathrm{~km} \mathrm{~s}^{-1}$ was measured for $\mathrm{H} \alpha$ and $\mathrm{H} \beta$ suggesting expansion velocity of around $1800 \mathrm{~km} \mathrm{~s}^{-1}$. This moderately high expansion velocity, as well as the broad, jagged profiles of the Balmer emission lines (see section 6.6.1), are features of the $\mathrm{He} / \mathrm{N}$ class that is characterized by a HWZI larger than $2500 \mathrm{~km}$ $\mathrm{s}^{-1}$ Williams 1992). The large HWZI values for the He/N novae are due to high ejecta velocities, these arise from the initial explosion on the WD. Before the nebular phase of nova SMCN 2016-10a, we note similarity with the spectrum of KT Eridani, also a very fast $\mathrm{He} / \mathrm{N}$ nova. However, when KT Eridani enters the nebular phase the blended lines of N III 4638 and HeII 4686 split and a narrow HeII emerges and grows becoming stronger than $\mathrm{H} \beta$ (see figure 2 in Imamura \& Tanabe 2012 which is not the case for nova SMCN 2016-10a.

In the spectra of day 20 to 23 , the forbidden nebular [O III] transitions at $4363 \AA$, $4959 \AA$ and $5007 \AA$ emerge while the nitrogen lines start to fade. The blending of the [O III] $5007 \AA$ with the N II $5001 \AA$ gives an artificially high [O III $5007 / 4959$ flux ratio at this stage. This flux ratio and the present emission lines indicate that the nova is in transition and had started to enter the optically thin nebular phase by the end of October leading to a correct prediction of the imminent SSS turn-on (Darnley \& Williams 2016). These forbidden lines are expected to initially emerge from low density gas due to the expansion of the nova (Williams 1992).

The spectra of day 27 and 32 show that the nova had entered the nebular phase with the He II $4686 \AA$ line becoming stronger than $\mathrm{H} \beta$. According to Williams (1992), He/N novae are expected to evolve in three distinct ways in the nebular stage: either showing no forbidden lines while the emission spectra fade into the continuum; coronal lines emerge (e.g. [Fex] $6375 \AA$ ); or the third scenario, where strong forbidden Ne lines emerge resulting in a Ne nova. The spectra of day 32 (Figure 6) show relatively strong [Ne v] lines compared to the Balmer and [O III], where the strength of [Ne V] $3426 \AA$ is comparable to [O III] $5007 \AA$. Some novae show very strong Ne emission, and these novae are considered Ne novae. For these novae, the Ne abundance exceeds solar abundance. Without a detailed calculation of element abundances, it is difficult to determine if the progenitor of nova SMCN 2016-10 contains a Ne WD. According to stellar evolution theory, Ne novae are expected to originate on a high mass WD (close to Chandrasekhar mass, $M_{\mathrm{WD}} \geqslant$ $1.08 \mathrm{M}_{\odot}$; see e. g. Nomoto 1984, Truran \& Livio 1986, Hurley, Pols \& Tout 2000 and references therein). It is worth noting that Shara \& Prialnik (1994), showed that it is possible to form a moderate-mass $\mathrm{Ne}-\mathrm{Mg}$ rich $\mathrm{WD}$, due to a high accretion rate of hydrogen onto the surface of a $\mathrm{C}-\mathrm{O}$ WD prior to the nova eruption, resulting in a massive $\mathrm{Ne}-$ $\mathrm{Mg}$ rich layer on the surface of the WD (see also Hachisu \& Kato 2016a). It is worth noting that the evolution of $\mathrm{H} \gamma$ is strongly affected by the [O III] $4363 \AA$ line from day 27 and that the evolution of $\mathrm{H} \delta$ is affected by instrumental noise. The late spectra of day 275 shows [O III] lines and $\mathrm{H} \alpha$ with the absence of forbidden Ne lines.

\subsection{Line profile evolution}

\subsubsection{Balmer lines}

The Balmer lines in the SALT high resolution spectra showed asymmetric profiles soon after maximum light (Figure 22. On day 8, all the Balmer lines showed a red-shifted - relative to the rest wavelength - single-emission peak at around $+1200 \mathrm{~km} \mathrm{~s}^{-1}$. This single emission peak might be due to an inclined (relative to the observer) asymmetric ejecta. Aspherical ejecta might also explain the difference between the positive (red) and negative (blue) sides of the line profiles. The relative strength of this emission feature decreases passing from $\mathrm{H} \alpha$ to $\mathrm{H} \delta$. Faint absorption features are observed for $\mathrm{H} \beta, \mathrm{H} \gamma$, and $\mathrm{H} \delta$. Such absorption features were also observed in the same lines of nova V339 Delphini, also a fast nova (Burlak et al. 2015) that formed dust at some point. For V339 Del, the hydrogen Balmer lines were initially flat-topped and later developed a more "peaked" profile, which is opposite to the evolution of nova SMCN 2016-10a. In the latter case, the peak at $\sim+1200 \mathrm{~km} \mathrm{~s}^{-1}$ faded and the lines show jagged, flat-topped profiles. These jagged, flat-topped profiles are probably an indication of clumpiness in the ejecta, which might lead to a disordered magnetic field and therefore reduce the intrinsic polarization (see Section 3.6. They might also be associated with 

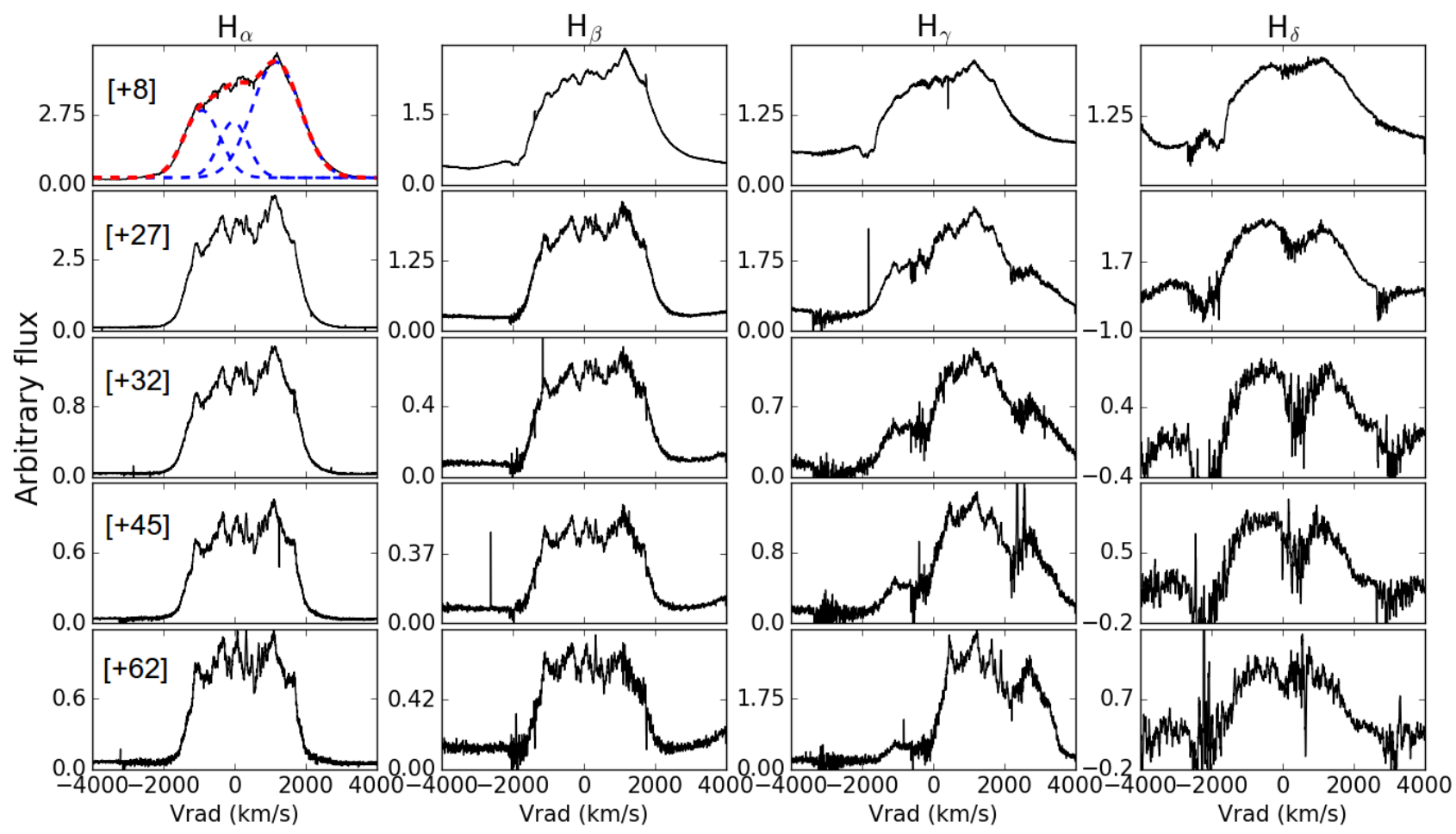

Figure 22. The evolution of Balmer lines from SALT high resolution spectra. From left to right: $\mathrm{H} \alpha, \mathrm{H} \beta, \mathrm{H} \gamma$, and $\mathrm{H} \delta$. The numbers between brackets are days after $t_{0}$. Heliocentric correction is applied to the radial velocities. The top-left panel shows a sample of multiple Gaussian fitting to the $\mathrm{H} \alpha$ line used to derive the FWHM.

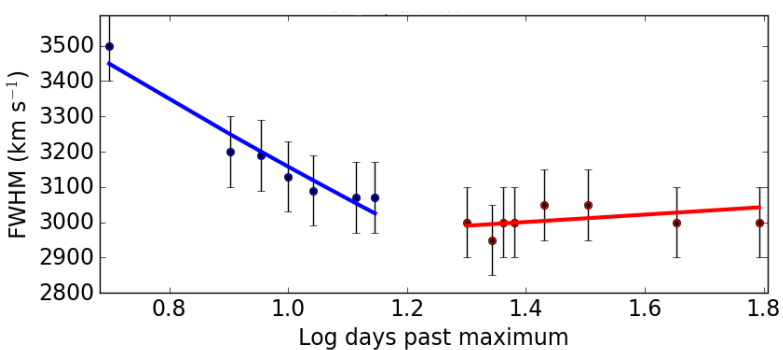

Figure 23. The FWHM of $\mathrm{H} \alpha$ plotted against the log time in days since maximum light. The blue circles represent the FWHM before day 14 and the red circles represent the FWHM from day 20 onward. A power law was fitted to the evolution of the FWHM of $\mathrm{H} \alpha$ from day 5 to day 14 resulting in an exponent of $\sim-0.14$.

the complete envelope ejection at the end of the wind phase after the eruption (Della Valle et al. 2002). The evolution of the lines also resemble that of nova V959 Mon (nova Mon 2012) in Shore et al. (2013). Nova V959 Mon is a slow ONe nova and it was the first nova to be discovered in the $\gamma$-rays prior to optical detection due to its proximity to the Sun during eruption. The Balmer lines of Nova V959 Mon show a red-shifted single emission peak with jagged profiles that evolved into more flat-topped profiles with time (see figure 2 in Shore et al. 2013).

The FWHM of $\mathrm{H} \alpha$ and $\mathrm{H} \beta$ were derived by applying multiple component Gaussian fitting in the IRAF and python environments separately (see Figure 22, The results are shown in Table 1 The FWHM of both lines shows a de- creasing trend and a systematic narrowing of the lines might be due to the deceleration of the ejecta after the eruption. The evolution of the FWHM is presented in Figure 23. This evolution follows a decreasing trend of a power law in time with an exponent $\mathrm{n} \sim-0.14\left(\Delta t^{n}\right)$ where $\Delta t$ is equal to the time since maximum light. Other novae have shown similar line narrowing after the eruption (see e.g. Della Valle et al. 2002 Hatzidimitriou et al. 2007, Shore et al. 2013|| Darnley et al. 2016).

Bode \& Kahn (1985) carried out modelling for the 1985 eruption of RS Ophiuchi and they suggested a three-phase shock model, where the high-velocity ejecta interact with the low-velocity stellar wind from the companion, which is a red giant in the case of RS Ophiuchi. The interaction between the ejecta and the wind generates shocks that are responsible for the ejecta deceleration, and hence the line narrowing.

Another explanation for this line narrowing was proposed by Shore et al. (1996), who suggested that it is due to the distribution of the matter velocity at the ejection moment. The emissivity of the fastest moving gas decreases with time at a higher rate than the slower moving gas, due to the decrease of its density, leading to the line narrowing.

After a survey of novae in M31, Shafter et al. (2011) carried out spectroscopic and photometric analysis of 91 novae and derived a relation between the FWHM of $\mathrm{H} \alpha$ and the light-curve parameter $t_{2}$ showing a clear trend of faster novae exhibiting higher expansion velocities. Although, the study is conducted in a different galaxy than the SMC, which means different metallicity and Hubble type, we ap- 
Table 1. The FWHM of $\mathrm{H} \alpha$ and $\mathrm{H} \beta$ at each observation. The values are in $\mathrm{km} \mathrm{s}^{-1}$.

\begin{tabular}{rcr}
\hline$\Delta t=t-t_{0}$ \\
(days) & $\begin{array}{r}\text { FWHM }(\mathrm{H} \alpha) \\
\pm 100\left(\mathrm{~km} \mathrm{~s}^{-1}\right)\end{array}$ \\
\hline 5 & 3500 & 3450 \\
8 & 3200 & 3050 \\
9 & 3190 & 3050 \\
10 & 3130 & 3000 \\
11 & 3090 & 2850 \\
13 & 3070 & 2950 \\
14 & 3070 & - \\
20 & 3000 & 3150 \\
22 & 2950 & 2900 \\
23 & 3000 & 2850 \\
24 & 3000 & 2900 \\
27 & 3050 & 3000 \\
32 & 3050 & 2950 \\
45 & 3000 & 2850 \\
62 & 3000 & 2800 \\
\hline
\end{tabular}

plied their relation to this nova:

$\log t_{2}(d)=6.84 \pm 0.1-(1.68 \pm 0.02) \times \log \left[\mathrm{FWHM}_{\mathrm{H} \alpha}\left(\mathrm{km} \mathrm{s}^{-1}\right)\right]$, with a FWHM of $\sim 3500 \pm 100 \mathrm{~km} \mathrm{~s}^{-1}$, leading to $t_{2}=$ $7.6 \pm 2$ days which is slightly larger than the values derived from our photometric data (see section 2.3).

In order to check for the blending of the [N II] $6548 \AA$ and $6583 \AA$ lines with $\mathrm{H} \alpha$ in terms of an apparent extra emission component in radial velocity, we directly compared the evolution of $\mathrm{H} \alpha$ and $\mathrm{H} \beta$ (Figure 24). Although the lines are very broad, no evidence of blending is present in any of the observations. If present, the [N II] $6548 \AA$ is at $\sim$ $600 \mathrm{~km} \mathrm{~s}^{-1}$ to the blue side of $\mathrm{H} \alpha$ and $[\mathrm{N} \mathrm{II}] 6583 \AA$ is at $\sim+$ $1000 \mathrm{~km} \mathrm{~s}^{-1}$ to the red side of $\mathrm{H} \alpha$. The line profile structure and evolution of $\mathrm{H} \alpha$ and $\mathrm{H} \beta$ is almost identical in the five high resolution observations. $\mathrm{H} \alpha$ does not show any extra emission at these two velocities in comparison to $\mathrm{H} \beta$. The peak at $\sim+1200 \mathrm{kms}^{-1}$ is present in both lines, as well in $\mathrm{H} \delta$ and $\mathrm{H} \gamma$ as discussed previously.

\subsubsection{Oxygen lines}

The nebular [O III] $4363 \AA, 4959 \AA$, and $5007 \AA$ lines were observed in all the spectra from day 20 onwards. The lines were absent in the first two spectra (day 5 and 8) since the nova was still close to maximum light and only entered the nebular stage in late October. The [O III] $4363 \AA$ is blended with $\mathrm{H} \gamma$. We show the evolution of the [O III] $5007 \AA$ line in Figure 25

Williams (2012) showed that the O I 8446/7773 intensity ratio is a good indicator of the density of gas that forms the spectrum during early decline. Fe II spectra are expected to have a high intensity $7773 \AA$ line, which is thought to originate from high density gas that persists for months after the eruption. Hence, the O I 8446/7773 ratio is low for the Fe II spectra (sometimes lower than 2), and sometimes showing small changes over months time. For the He/N class, the lines are expected to originate from low density gas where the line at $7773 \AA$ is sometimes not even detected. Hence, the ratio of the two oxygen lines should be high, followed by a fast decrease. We derived the flux ratio of O i 8446/7773

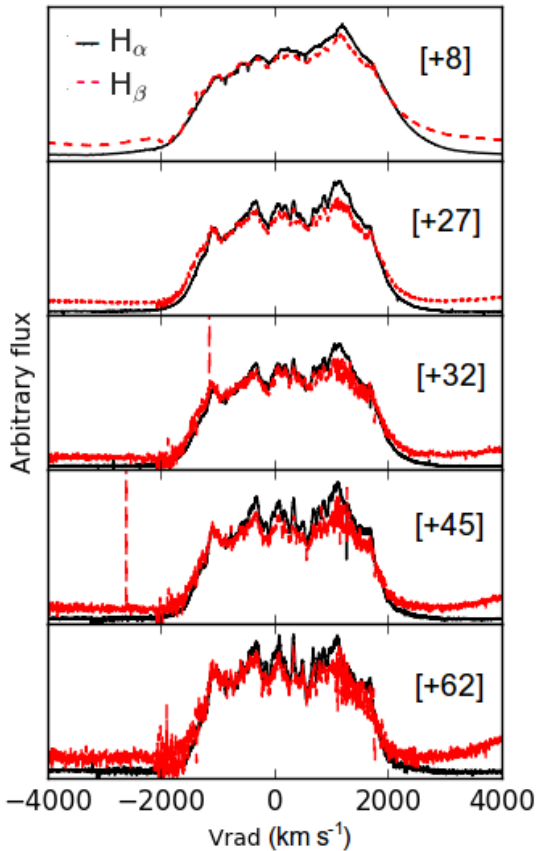

Figure 24. The evolution of $\mathrm{H} \alpha$ (solid black) and $\mathrm{H} \beta$ (dashed red) line profiles. The numbers between brackets are days since $t_{0}$.

Table 2. The flux ratio of O I 8446/7773 from the HRS and RSS observations. The flux is not corrected for reddening.

\begin{tabular}{rr}
\hline$\Delta t=t-t_{0}$ & $F($ O I $8446 / 7773)$ \\
(days) & \\
\hline 5 & 2.3 \\
8 & 3.6 \\
23 & 5.5 \\
27 & 5.4 \\
32 & 4.5 \\
45 & 2.3 \\
62 & 1.2 \\
\hline
\end{tabular}

for all our observations and the results are shown in Table 2. They show a high increasing ratio followed by a fast decrease, as expected for a $\mathrm{He} / \mathrm{N}$ nova.

\section{SUMMARY AND CONCLUSIONS}

Nova SMCN 2016-10a was discovered on 2016-10-14.19 UT by MASTER-OAFA auto-detection system (Shumkov et al. 2016). Pre-discovery observations from MASTER (Lipunov et al. 2016) and a DSLR camera from Sao Jose dos Campos, Brazil (Jablonski \& Oliveira 2016), allowed us to estimate $t_{0}$ as 2016-10-09.2 UT and the date of maximum optical light as 2016-10-09.8 UT. Nova SMCN 2016-10a is the best studied nova in the SMC so far. The optical, NIR, X-ray, and UV data, led us to the following conclusions:

1. Nova SMCN 2016-10a is a very fast nova with $t_{2} \simeq$ $4.0 \pm 1.0 \mathrm{~d}$. This is an indication of an eruption that occurred on a high mass WD $\left(M_{\mathrm{WD}} \geqslant 1.25 \mathrm{M}_{\odot}\right)$.

2. The light-curve is consistent with a nova at the distance 


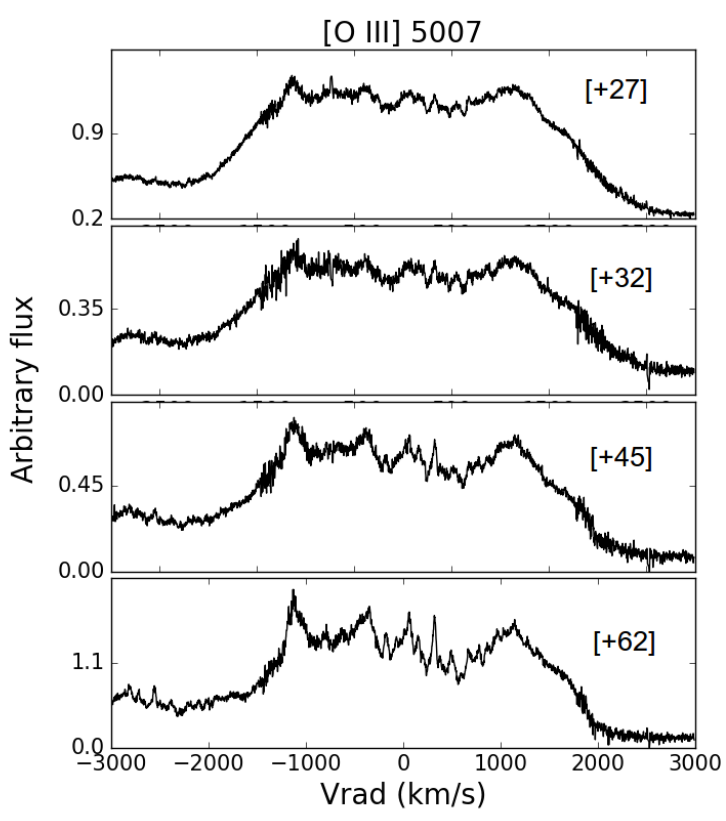

Figure 25. The evolution of [O III] $5007 \AA$ line profiles. The rise to the blue side of the line is due to the [O III] $4959 \AA$ line. The numbers between brackets are days after $t_{0}$. Heliocentric correction is applied to the radial velocities.

of the SMC, although its magnitudes at 15 and 17 days indicate it may be to the fore of the bulk of the SMC.

3. At a distance of $61 \pm 10 \mathrm{kpc}$, it is the brightest nova discovered in the cloud with $M_{V \text {, max }} \approx-10.5 \pm 0.5$ and probably one of the brightest on record.

4. Based on the photometric data from the quiescence phase and the early decline phase, we suggest that the progenitor system is likely to contain a main sequence secondary, or a sub-giant secondary.

5. Based on the optical spectra, we classified nova SMCN 2016-10a as a He/N nova indicating a system with a high mass WD.

6. After 5 days from optical peak, we measured FWHM of $\sim 3500 \pm 100 \mathrm{~km} \mathrm{~s}^{-1}$ from the $\mathrm{H} \alpha$ and $\mathrm{H} \beta$ lines, indicating moderately high expansion velocities.

7. The light-curve during quiescence, the Balmer line profiles, as well as the negligible polarization, suggest the system might be at a low inclination and that there are clumps in the ejecta. Line modelling is definitely needed to give us a better insight into the structure and morphology of the system and the ejecta.

8. The high temperature $(\sim 90 \mathrm{eV})$ of the super-soft X-ray emission, together with the relatively rapid turn-on and -off times ( $28 \mathrm{~d}$ and $180 \mathrm{~d}$, respectively), suggest a high mass WD $\left(\sim 1.25-1.3 \mathrm{M}_{\odot}\right)$, in agreement with the results from the optical data. We note that, at a distance of $61 \mathrm{kpc}$, the bolometric luminosity is a factor of 1.76 higher than at $46 \mathrm{kpc}$.

9. The plateaux in the UV light-curves extend at least from day $\sim 90$ to 170 , approximately corresponding to the interval of bright, high temperature soft X-rays.

10. The UV line fluxes show different light-curves before day 20 suggesting optical thickness is the cause. The line fluxes have the same light-curves in the later data, suggesting the ejecta are effectively optically thin by then, and that is also when we see the rise of the X-ray flux.

Once the system returns to quiescence, more observations are essential to constrain the type of the secondary star. If it becomes possible to measure the expansion parallax of this nova then the question of its brightness and location could be settled. Such measurements are not easy and often raise questions about the symmetry of the eruption. With the available spectroscopic data, line modelling is encouraged to follow-up this work, which can help to constrain the structure and morphology of the system.

\section{ACKNOWLEDGMENTS}

A part of this work is based on observations made with the Southern African Large Telescope (SALT), under the program 2016-1-MLT-010 and 2016-2-LSP-001. EA, DB, PAW, SM, PW gratefully acknowledge the receipt of research grants from the National Research Foundation (NRF) of South Africa. We are grateful to Steve Crawford, Itumeleng Monageng, Daniel Viljoen, and Brent Miszalski for assistance with the SALT observations

AK acknowledges the National Research Foundation of South Africa and the Russian Science Foundation (project no.14-50-00043).

P.M. is supported by the "Diamond Grant" No. DI2013/014743 funded by the Polish Ministry of Science and Higher Education.

The OGLE project has received funding from the National Science Center, Poland, grant MAESTRO 2014/14/A/ST9/00121 to A.U.

KLP, NPMK, APB and JPO acknowledge support from the UK Space Agency.

FMW acknowledges support from the US National Science Foundation, grant 1614113. Based in part on observations from the SMARTS Observatory, which is hosted by the Cerro Tololo Inter American Observatory, National Optical Astronomy Observatory, which is operated by the Association of Universities for Research in Astronomy (AURA) under a cooperative agreement with the National Science Foundation. We thank the SMARTS queue schedulers and observers for their efforts.

Based on observations obtained at the Southern Astrophysical Research (SOAR) telescope, which is a joint project of the Ministério da Ciência, Tecnologia, e Inovação (MCTI) da República Federativa do Brasil, the U.S. National Optical Astronomy Observatory (NOAO), the University of North Carolina at Chapel Hill (UNC), and Michigan State University (MSU).

VARMR acknowledges financial support from FCT in the form of an exploratory project of reference IF/00498/2015, from CIDMA strategic project UID/MAT/04106/2013 and supported by Enabling Green E-science for the Square Kilometer Array Research Infrastructure (ENGAGE SKA), POCI-01-0145-FEDER-022217, funded by Programa Operacional Competitividade e Internacionalização (COMPETE 2020) and FCT, Portugal.

We thank Ulisse Munari and an anonymous referee for useful comments. 


\section{REFERENCES}

Arnaud K. A., 1996, in Astronomical Society of the Pacific Conference Series, Vol. 101, Astronomical Data Analysis Software and Systems V, Jacoby G. H., Barnes J., eds., p. 17

Ballester P., 1992, in European Southern Observatory Conference and Workshop Proceedings, Vol. 41, European Southern Observatory Conference and Workshop Proceedings, Grosbøl P. J., de Ruijsscher R. C. E., eds., p. 177

Balman Ş., Krautter J., Ögelman H., 1998, ApJ, 499, 395

Barnes S. I. et al., 2008, in Proc. SPIE, Vol. 7014, Groundbased and Airborne Instrumentation for Astronomy II, p. 70140K

Bath G. T., Harkness R. P., 1989, in Classical Novae, Bode M. F., Evans A., eds., pp. 61-72

Beardmore A. P. et al., 2010, The Astronomer's Telegram, 2423

Beardmore A. P. et al., 2012, A\&A, 545, A116

Bianchi L., Herald J., Efremova B., Girardi L., Zabot A., Marigo P., Conti A., Shiao B., 2011, Ap\&SS, 335, 161

Bode M. F. et al., 2016, ApJ, 818, 145

Bode M. F., Evans A., 2008, Classical Novae

Bode M. F., Kahn F. D., 1985, MNRAS, 217, 205

Booth R. A., Mohamed S., Podsiadlowski P., 2016, MNRAS, 457, 822

Bramall D. G. et al., 2012, in Proc. SPIE, Vol. 8446, Ground-based and Airborne Instrumentation for Astronomy IV, p. 84460A

Bramall D. G. et al., 2010, in Proc. SPIE, Vol. 7735, Ground-based and Airborne Instrumentation for Astronomy III, p. $77354 \mathrm{~F}$

Brandi E., Quiroga C., Mikołajewska J., Ferrer O. E., García L. G., 2009, A\&A, 497, 815

Brown T. M. et al., 2013, PASP, 125, 1031

Buckley D. A. H., Swart G. P., Meiring J. G., 2006, in Proc. SPIE, Vol. 6267, Society of Photo-Optical Instrumentation Engineers (SPIE) Conference Series, p. 62670Z

Burgh E. B., Nordsieck K. H., Kobulnicky H. A., Williams T. B., O'Donoghue D., Smith M. P., Percival J. W., 2003, in Society of Photo-Optical Instrumentation Engineers (SPIE) Conference Series, Vol. 4841, Instrument Design and Performance for Optical/Infrared Ground-based Telescopes, Iye M., Moorwood A. F. M., eds., pp. 1463-1471

Burlak M. A., Esipov V. F., Komissarova G. V., Shenavrin V. I., Taranova O. G., Tatarnikov A. M., Tatarnikova A. A., 2015, Baltic Astronomy, 24, 109

Burrows D. N. et al., 2005, Space Sci. Rev., 120, 165

Buscombe W., de Vaucouleurs G., 1955, The Observatory, 75, 170

Caldwell J. A. R., Coulson I. M., 1986, MNRAS, 218, 223

Cao Y. et al., 2012, ApJ, 752, 133

Cash W., 1979, ApJ, 228, 939

Clemens J. C., Crain J. A., Anderson R., 2004, in Proc. SPIE, Vol. 5492, Ground-based Instrumentation for Astronomy, Moorwood A. F. M., Iye M., eds., pp. 331-340 Coe M. J. et al., 2011, MNRAS, 414, 3281

Cohen J. G., 1985, ApJ, 292, 90

Crause L. A. et al., 2014, in Proc. SPIE, Vol. 9147, Groundbased and Airborne Instrumentation for Astronomy V, p. $91476 \mathrm{~T}$
Crawford S. M. et al., 2010, in Society of Photo-Optical Instrumentation Engineers (SPIE) Conference Series, Vol. 7737, Society of Photo-Optical Instrumentation Engineers (SPIE) Conference Series, p. 25

Darnley M. J. et al., 2006, MNRAS, 369, 257

Darnley M. J. et al., 2016, ApJ, 833, 149

Darnley M. J., Ribeiro V. A. R. M., Bode M. F., Hounsell

R. A., Williams R. P., 2012, ApJ, 746, 61

Darnley M. J., Williams S. C., 2016, The Astronomer's Telegram, 9688

De Gennaro Aquino I. et al., 2015, A\&A, 581, A134

Della Valle M., Livio M., 1995, ApJ, 452, 704

Della Valle M., Pasquini L., Daou D., Williams R. E., 2002, A\&A, 390, 155

Della Valle M. D., 2002, in American Institute of Physics Conference Series, Vol. 637, Classical Nova Explosions, Hernanz M., José J., eds., pp. 443-456

Downes R. A., Duerbeck H. W., 2000, AJ, 120, 2007

Ferrarese L., Côté P., Jordán A., 2003, ApJ, 599, 1302

Gallagher J. S., Ney E. P., 1976, ApJ, 204, L35

Gallagher J. S., Starrfield S., 1978, ARA\&A, 16, 171

Gaposchkin C. H. P., 1957, The galactic novae.

Gehrels N. et al., 2004, ApJ, 611, 1005

Gorbovskoy E. et al., 2010, Advances in Astronomy, 2010, 917584

Graham J. A., 1979, in IAU Colloq. 46: Changing Trends in Variable Star Research, Bateson F. M., Smak J., Urch I. H., eds., Vol. 46, p. 96

Hachisu I., Kato M., 2006, ApJS, 167, 59

Hachisu I., Kato M., 2014, ApJ, 785, 97

Hachisu I., Kato M., 2016a, ApJ, 816, 26

Hachisu I., Kato M., 2016b, ApJS, 223, 21

Haschke R., Grebel E. K., Duffau S., 2011, AJ, 141, 158

Haschke R., Grebel E. K., Duffau S., 2012, AJ, 144, 107

Hatzidimitriou D., Reig P., Manousakis A., Pietsch W., Burwitz V., Papamastorakis I., 2007, A\&A, 464, 1075

Henden A. A., Welch D. L., Terrell D., Levine S. E., 2009, in American Astronomical Society Meeting Abstracts, Vol. 214, American Astronomical Society Meeting Abstracts \#214, p. 669

Henze M. et al., 2014, A\&A, 563, A2

Hillman Y., Prialnik D., Kovetz A., Shara M. M., 2016, ApJ, 819, 168

Hillman Y., Prialnik D., Kovetz A., Shara M. M., Neill J. D., 2014, MNRAS, 437, 1962

Hounsell R. et al., 2010, ApJ, 724, 480

Humphrey P. J., Liu W., Buote D. A., 2009, ApJ, 693, 822

Hurley J. R., Pols O. R., Tout C. A., 2000, MNRAS, 315, 543

Ikeda Y., Kawabata K. S., Akitaya H., 2000, A\&A, 355, 256

Imamura K., Tanabe K., 2012, PASJ, 64, 120

Jablonski F., Oliveira A., 2016, The Astronomer's Telegram, 9684

Jack D. et al., 2017, ArXiv e-prints

Jacoby G. H. et al., 1992, PASP, 104, 599

Jacyszyn-Dobrzeniecka A. M. et al., 2016, Acta Astron., 66, 149

Kasliwal M. M. et al., 2011, ApJ, 730, 134

Kato M., Hachisu I., Luna G. J. M., 2008, in Astronomical Society of the Pacific Conference Series, Vol. 401, RS Ophiuchi (2006) and the Recurrent Nova Phenomenon, 
Evans A., Bode M. F., O’Brien T. J., Darnley M. J., eds., p. 308

Kniazev A. Y., Gvaramadze V. V., Berdnikov L. N., 2016, MNRAS, 459, 3068

Kobulnicky H. A., Nordsieck K. H., Burgh E. B., Smith M. P., Percival J. W., Williams T. B., O'Donoghue D., 2003, in Society of Photo-Optical Instrumentation Engineers (SPIE) Conference Series, Vol. 4841, Instrument Design and Performance for Optical/Infrared Ground-based Telescopes, Iye M., Moorwood A. F. M., eds., pp. 16341644

Krautter J., Oegelman H., Starrfield S., Wichmann R., Pfeffermann E., 1996, ApJ, 456, 788

Kuin N. P. M. et al., 2015, MNRAS, 449, 2514

Kuin N. P. M., Page K. L., Williams S. C., Darnley M. J., Shore S. N., Walter F. M., 2016, The Astronomer's Telegram, 9635

Kuin P., 2014, UVOTPY: Swift UVOT grism data reduction. Astrophysics Source Code Library

Lipunov V. et al., 2016, The Astronomer's Telegram, 9631 Livio M., 1992, ApJ, 393, 516

MacDonald J., 1996, in Astrophysics and Space Science Library, Vol. 208, IAU Colloq. 158: Cataclysmic Variables and Related Objects, Evans A., Wood J. H., eds., p. 281

Mason E., Della Valle M., Gilmozzi R., Lo Curto G., Williams R. E., 2005, A\&A, 435, 1031

McLaughlin D. B., 1939, Popular Astronomy, 47, 481

McLaughlin D. B., 1944, Popular Astronomy, 52, 109

Monet D. G. et al., 2003, AJ, 125, 984

Moore C. E., 1945, Contributions from the Princeton University Observatory, 20, 1

Mroz P., Udalski A., 2016, The Astronomer's Telegram, 9622

Mróz P. et al., 2016, ApJS, 222, 9

Munari U., Hambsch F.-J., Frigo A., 2017, ArXiv e-prints

Munari U., Zwitter T., 1997, A\&A, 318, 269

Ness J.-U. et al., 2015, A\&A, 578, A39

Ness J.-U. et al., 2009, AJ, 137, 4160

Neugent K. F., Massey P., Skiff B., Drout M. R., Meynet G., Olsen K. A. G., 2010, ApJ, 719, 1784

Nomoto K., 1984, ApJ, 277, 791

Nordsieck K. H., Jaehnig K. P., Burgh E. B., Kobulnicky H. A., Percival J. W., Smith M. P., 2003, in Proc. SPIE, Vol. 4843, Polarimetry in Astronomy, Fineschi S., ed., pp. 170-179

Ochsenbein F., Bauer P., Marcout J., 2000, A\&AS, 143, 23

O'Donoghue D. et al., 2006, MNRAS, 372, 151

Osborne J. P., 2015, Journal of High Energy Astrophysics, 7,117

Osborne J. P. et al., 2011, ApJ, 727, 124

Page K. L., 2013, in IAU Symposium, Vol. 281, Binary Paths to Type Ia Supernovae Explosions, Di Stefano R., Orio M., Moe M., eds., pp. 96-104

Page K. L., Kuin N. P., Osborne J. P., Schwarz G. J., Shore S. N., Starrfield S., Woodward C. E., 2014, The Astronomer's Telegram, 5967

Page K. L., Osborne J. P., 2014, in Astronomical Society of the Pacific Conference Series, Vol. 490, Stellar Novae: Past and Future Decades, Woudt P. A., Ribeiro V. A. R. M., eds., p. 345

Page K. L. et al., 2010, MNRAS, 401, 121

Page K. L. et al., 2015, MNRAS, 454, 3108
Page K. L., Walter F. M., Schwarz G. J., Osborne J. P., 2013a, The Astronomer's Telegram, 4853

Page M. J. et al., 2013b, MNRAS, 436, 1684

Payne-Gaposchkin C., 1964, The galactic novae

Perryman M. A. C., 1997, in ESA Special Publication, Vol. 402, Hipparcos - Venice '97, Bonnet R. M., Høg E., Bernacca P. L., Emiliani L., Blaauw A., Turon C., Kovalevsky J., Lindegren L., Hassan H., Bouffard M., Strim B., Heger D., Perryman M. A. C., Woltjer L., eds., pp. 1-4

Pietsch W., 2010, Astronomische Nachrichten, 331, 187

Poole T. S. et al., 2008, MNRAS, 383, 627

Poznanski D., Prochaska J. X., Bloom J. S., 2012, MNRAS, 426, 1465

Ribeiro V. A. R. M., Bode M. F., Darnley M. J., Barnsley R. M., Munari U., Harman D. J., 2013, MNRAS, 433, 1991

Ribeiro V. A. R. M., Munari U., Valisa P., 2013, ApJ, 768, 49

Rodrigues C. V., Magalhães A. M., Coyne G. V., Piirola S. J. V., 1997, ApJ, 485, 618

Roming P. W. A. et al., 2005, Space Sci. Rev., 120, 95

Sala G., Hernanz M., 2005, A\&A, 439, 1061

Saxton C. J., Soria R., Wu K., Kuin N. P. M., 2012, MNRAS, 422, 1625

Schaefer B. E., 2010, ApJS, 187, 275

Schwarz G. J. et al., 2011, ApJS, 197, 31

Schwarz G. J. et al., 2015, AJ, 149, 95

Serkowski K., Mathewson D. S., Ford V. L., 1975, ApJ, 196, 261

Shafter A. W., 1997, ApJ, 487, 226

Shafter A. W. et al., 2011, ApJ, 734, 12

Shafter A. W., Rau A., Quimby R. M., Kasliwal M. M., Bode M. F., Darnley M. J., Misselt K. A., 2009, ApJ, 690, 1148

Shappee B. J. et al., 2014, ApJ, 788, 48

Shara M. M. et al., 2017a, ArXiv e-prints - 1702.05788v1

Shara M. M. et al., 2017b, ArXiv e-prints - 1702.06988v2

Shara M. M., Prialnik D., 1994, AJ, 107, 1542

Shore S. N., Augusteijn T., Ederoclite A., Uthas H., 2011, A\&A, 533, L8

Shore S. N., De Gennaro Aquino I., Schwarz G. J., Augusteijn T., Cheung C. C., Walter F. M., Starrfield S., 2013, A\&A, 553, A123

Shore S. N., Kenyon S. J., Starrfield S., Sonneborn G., 1996, ApJ, 456, 717

Shore S. N. et al., 2016, A\&A, 590, A123

Shumkov V. et al., 2016, The Astronomer's Telegram, 9621 Soraisam M. D., Gilfanov M., Wolf W. M., Bildsten L., 2016, MNRAS, 455, 668

Stahl O., Kaufer A., Tubbesing S., 1999, in Astronomical Society of the Pacific Conference Series, Vol. 188, Optical and Infrared Spectroscopy of Circumstellar Matter, Guenther E., Stecklum B., Klose S., eds., p. 331

Stockman H. S., Schmidt G. D., Lamb D. Q., 1988, ApJ, 332,282

Strope R. J., Schaefer B. E., Henden A. A., 2010, AJ, 140, 34

Subramanian S. et al., 2017, MNRAS

Tody D., 1986, in Society of Photo-Optical Instrumentation Engineers (SPIE) Conference Series, Vol. 627, Instrumentation in astronomy VI, Crawford D. L., ed., p. 733 
Truran J. W., Livio M., 1986, ApJ, 308, 721

Udalski A., Szymański M. K., Szymański G., 2015, Acta

Astron., 65, 1

Walter F. M., Battisti A., Towers S. E., Bond H. E., Stringfellow G. S., 2012, PASP, 124, 1057

Warner B., 1985, MNRAS, 217, 1P

Warner B., 1995a, Cambridge Astrophysics Series, 28

Warner B., 1995b, Cambridge Astrophysics Series, 28

Wenger M. et al., 2000, A\&AS, 143, 9

Wilking B. A., Lebofsky M. J., Kemp J. C., Martin P. G.,

Rieke G. H., 1980, ApJ, 235, 905

Williams R. E., 1992, AJ, 104, 725

Williams R. E., 1994, ApJ, 426, 279

Williams R. E., 2012, AJ, 144, 98

Williams S. C., Darnley M. J., 2016, The Astronomer's Telegram, 9628

Wolf W. M., Bildsten L., Brooks J., Paxton B., 2013, ApJ, 777,136

Yaron O., Prialnik D., Shara M. M., Kovetz A., 2005, ApJ, 623, 398

Young P. R., Feldman U., Lobel A., 2011, ApJS, 196, 23

Zaritsky D., Harris J., Thompson I. B., Grebel E. K., Massey P., 2002, AJ, 123, 855

\section{APPENDIX A: OBSERVATIONS LOG}

In this Appendix we list all the observations log. The OGLE, SMARTS, and Swift UVOT photometry can be found on the electronic version. The OGLE time series photometry are also available from the OGLE Internet Archive 6 The SMARTS time series photometry is available in the SMARTS atlas, at 7 In Table A9 we list the line IDs along with the FWHM, Equivalent Widths (EWs), and fluxes for those emission lines for which an estimate was possible.

${ }^{6}$ http://ogle.astrouw.edu.pl/ogle4/cvom/smcn-2016-10a.html

7 http://www.astro.sunysb.edu/fwalter/SMARTS/NovaAtlas/nsmc2016/nsmc2016.html 
Table A1. A sample of OGLE $V$-band photometry. The time series photometry is available from the OGLE Internet Archive and on the electronic version.

\begin{tabular}{rrrr}
\hline HJD & $\begin{array}{r}t-t_{0} \\
(\text { days })\end{array}$ & \multicolumn{2}{c}{$V$} \\
& $\begin{array}{r}\Delta V \\
(\mathrm{mag})\end{array}$ \\
\hline 2455399.88 & -2270.81 & 20.60 & 0.03 \\
2455434.82 & -2235.87 & 20.87 & 0.12 \\
2455446.70 & -2223.99 & 20.47 & 0.02 \\
2455456.81 & -2213.88 & 20.58 & 0.03 \\
2455486.62 & -2184.07 & 20.40 & 0.06 \\
2455499.63 & -2171.07 & 20.98 & 0.04 \\
2455503.62 & -2167.08 & 20.72 & 0.04 \\
2455516.60 & -2154.10 & 20.51 & 0.06 \\
2455536.58 & -2134.11 & 20.48 & 0.03 \\
\hline
\end{tabular}

Table A2. A sample of OGLE I-band photometry. The time series photometry is available from the OGLE Internet Archive and on the electronic version.

\begin{tabular}{rrrr}
\hline HJD & $\begin{array}{r}t-t_{0} \\
(\text { days })\end{array}$ & \multicolumn{2}{c}{$I$} \\
& $(\mathrm{mag})$ & $\Delta I$ \\
\hline 2455346.93 & -2323.77 & 20.14 & 0.20 \\
2455347.91 & -2322.79 & 20.42 & 0.23 \\
2455358.90 & -2311.80 & 20.06 & 0.13 \\
2455364.91 & -2305.79 & 20.35 & 0.13 \\
2455376.94 & -2293.76 & 20.09 & 0.13 \\
2455378.94 & -2291.76 & 20.40 & 0.17 \\
2455380.91 & -2289.79 & 20.35 & 0.20 \\
2455381.92 & -2288.79 & 20.44 & 0.21 \\
2455384.89 & -2285.81 & 20.49 & 0.20 \\
\hline
\end{tabular}

Table A3. The FLOYDS spectroscopic observations log.

\begin{tabular}{rrr}
\hline HJD & $\begin{array}{r}t-t_{0} \\
\text { (days) }\end{array}$ & $\begin{array}{r}\text { Exposure time } \\
(\mathrm{s})\end{array}$ \\
\hline 2457676.19 & 5.4 & $3 \times 60$ \\
2457679.10 & 8.3 & $3 \times 60$ \\
2457680.04 & 9.3 & $3 \times 60$ \\
2457681.20 & 10.5 & $6 \times 60$ \\
2457682.00 & 11.3 & $3 \times 120$ \\
2457684.12 & 13.4 & $2 \times 120$ \\
2457685.13 & 14.4 & $3 \times 120$ \\
2457691.11 & 20.4 & $3 \times 300$ \\
2457693.15 & 22.4 & $3 \times 300$ \\
2457695.07 & 24.3 & $3 \times 450$ \\
2457703.20 & 32.5 & $3 \times 450$
\end{tabular}

Table A4. A sample of SMARTS BVRIJHK photometry. The time series photometry is available on the electronic version and on the SMARTS atlas.

\begin{tabular}{rrrrr}
\hline HJD & $\begin{array}{r}\left(t-t_{0}\right) \\
(\text { days })\end{array}$ & Band & Magnitude & $\begin{array}{r}\text { Instrument } \\
\text { uncertainty }\end{array}$ \\
\hline 2457682.64 & 11.93 & $I$ & 10.84 & 0.002 \\
2457682.64 & 11.93 & $H$ & 10.21 & 0.030 \\
2457682.64 & 11.93 & $B$ & 11.73 & 0.001 \\
2457682.64 & 11.93 & $J$ & 9.83 & 0.037 \\
2457682.64 & 11.93 & $K$ & 9.95 & 0.033 \\
2457682.64 & 11.93 & $V$ & 12.14 & 0.002 \\
2457682.64 & 11.93 & $R$ & 10.64 & 0.001 \\
2457683.73 & 13.02 & $I$ & 10.90 & 0.002 \\
2457683.73 & 13.02 & $J$ & 9.95 & 0.038 \\
2457683.73 & 13.02 & $H$ & 10.45 & 0.034 \\
2457683.73 & 13.02 & $B$ & 11.57 & 0.001 \\
2457683.73 & 13.02 & $K$ & 10.05 & 0.039 \\
2457683.73 & 13.02 & $V$ & 12.07 & 0.002 \\
2457683.73 & 13.02 & $R$ & 10.67 & 0.001 \\
2457684.73 & 14.02 & $I$ & 11.10 & 0.002 \\
2457684.73 & 14.02 & $J$ & 10.74 & 0.043 \\
2457684.73 & 14.02 & $H$ & 10.653 & 0.036 \\
2457684.73 & 14.02 & $B$ & 11.73 & 0.001 \\
2457684.73 & 14.02 & $K$ & 10.02 & 0.04
\end{tabular}

Table A5. A sample of Swift UVOT uvw2 photometry. The time series photometry is available on the electronic version.

\begin{tabular}{|c|c|c|c|c|}
\hline HJD & $\begin{array}{r}t-t_{0} \\
\text { (days) }\end{array}$ & $\begin{array}{r}\text { exposure time } \\
(\mathrm{s})\end{array}$ & \multicolumn{2}{|c|}{ (mag) } \\
\hline 2457682.85 & 12.15 & 4.8 & 9.85 & 0.14 \\
\hline 2457682.86 & 12.16 & 4.8 & 9.68 & 0.21 \\
\hline 2457682.92 & 12.22 & 4.8 & 9.64 & 0.14 \\
\hline 2457682.93 & 12.23 & 16.4 & 9.76 & 0.18 \\
\hline 2457685.18 & 14.48 & 4.8 & 10.65 & 0.43 \\
\hline 2457685.18 & 14.48 & 15 & 10.12 & 0.15 \\
\hline 2457685.24 & 14.54 & 15 & 10.15 & 0.15 \\
\hline 2457685.25 & 14.55 & 10.7 & 9.93 & 0.215 \\
\hline 2457685.57 & 14.87 & 4.8 & 10.07 & 0.15 \\
\hline 2457685.58 & 14.88 & 17 & 10.06 & 0.19 \\
\hline
\end{tabular}

Table A6. A sample of Swift UVOT uvm 2 photometry. The time series photometry is available on the electronic version.

\begin{tabular}{|c|c|c|c|c|}
\hline HJD & $\begin{array}{l}t-t_{0} \\
\text { (days) }\end{array}$ & $\begin{array}{r}\text { exposure time } \\
\text { (s) }\end{array}$ & \multicolumn{2}{|c|}{ (mag) } \\
\hline 2457682.85 & 12.15 & 4.8 & 9.61 & 0.27 \\
\hline 2457682.85 & 12.15 & 50 & 9.91 & 0.15 \\
\hline 2457682.92 & 12.22 & 4.8 & 9.76 & 0.30 \\
\hline 2457685.18 & 14.48 & 4.8 & 10.12 & 0.15 \\
\hline 2457685.25 & 14.55 & 4.8 & 10.26 & 0.16 \\
\hline 2457685.57 & 14.87 & 4.8 & 10.32 & 0.03 \\
\hline 2457703.33 & 32.63 & 4.8 & 12.03 & 0.05 \\
\hline 2457703.33 & 32.63 & 46 & 12.07 & 0.03 \\
\hline
\end{tabular}




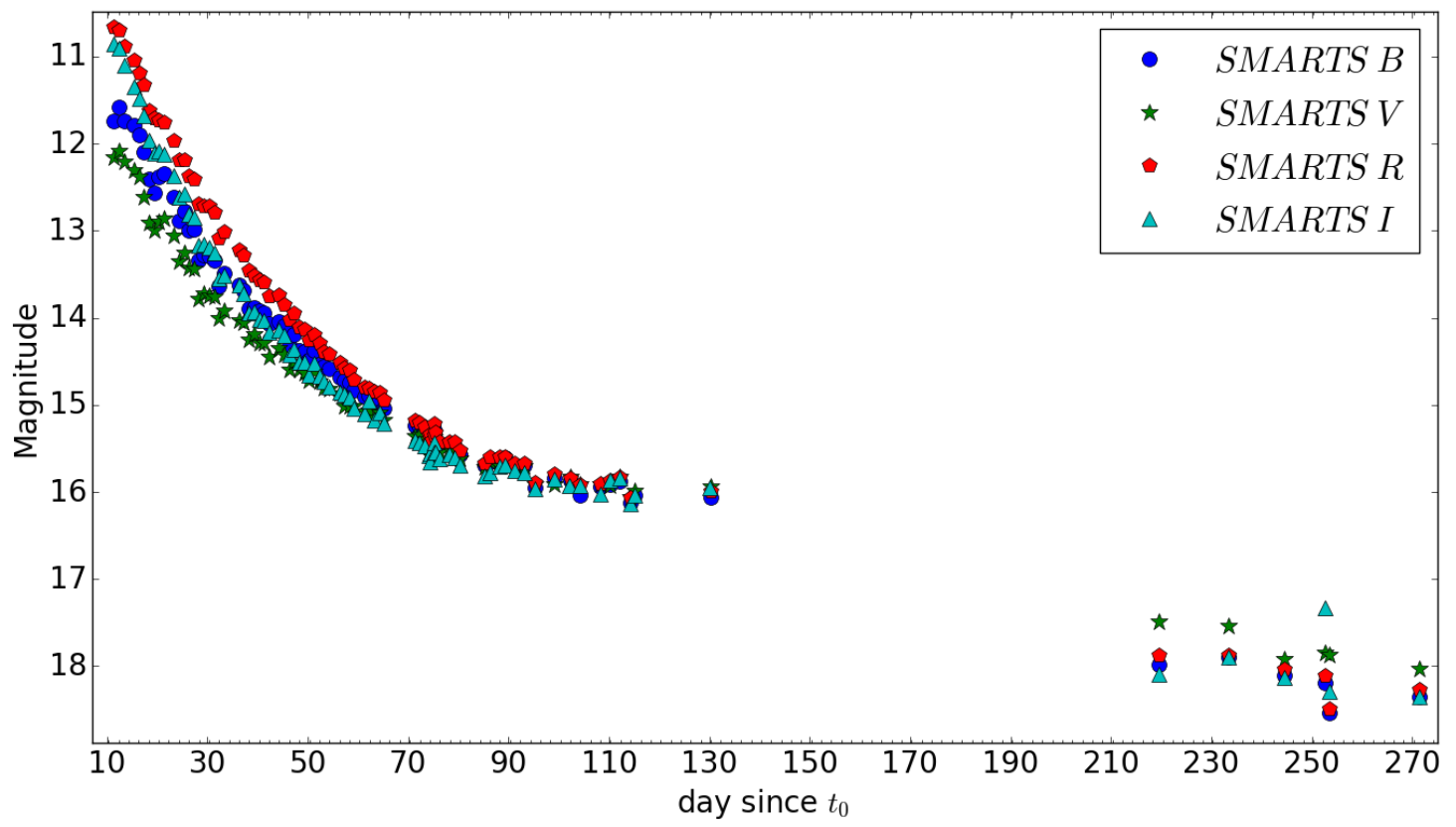

Figure A1. The full photometric $B V R I$ data from SMARTS as a function of days since eruption, colour and symbol coded as indicated in the legend. A colour version of this plot is present in the online journal.

Table A7. Summed Swift UV grism exposures

\begin{tabular}{rrrl}
\hline $\begin{array}{r}\text { HJD } \\
\text { (start })\end{array}$ & $\begin{array}{r}\text { HJD } \\
(\mathrm{end})\end{array}$ & $\begin{array}{r}<t-t_{0}> \\
(\text { days })\end{array}$ & quality notes ${ }^{1}$ \\
\hline 2457676.8036 & 2457678.7299 & 7.07 & 2ndO:2870-2950,3308-3390,3890-3980,4400-4500,5080-5150 \\
2457682.8616 & 2457685.2581 & 13.36 & Good 1700-4500,but rise above 4500-5060 \\
2457685.5775 & 2457688.7052 & 16.44 & Good 1700-5060,5060-6000 noisy and strange drop in flux \\
2457699.5597 & 2457705.4783 & 31.82 & ZOc:1831-1885;2ndO:2824-2945,3235-3375,3843-3947,4375-4530,5050-5170 \\
2457710.9143 & 2457714.0447 & 41.78 & Good 1700-4800, 4800-5100 shows rising continuum to red \\
2457715.6864 & 2457719.7391 & 47.01 & Good 1700-5200, then noisy \\
2457723.6584 & 2457734.8146 & 58.54 & Good 1700-5100 then noisy \\
2457762.1341 & 2457776.4872 & 98.61 & Continum level good to 3840 \\
2457788.4264 & 2457828.3827 & 137.70 & No contamination, bad S/N, very noisy for $>4100$ \\
\hline
\end{tabular}

1 2ndO: second order affects these ranges; ZOc: zeroth order contamination affects these ranges.

Table A8. The SALT spectroscopy and spectropolarimetry observations log.

\begin{tabular}{|c|c|c|c|c|}
\hline Type & HJD & $\begin{array}{r}t-t_{0} \\
\text { (days) }\end{array}$ & $\begin{array}{r}\text { Exposure time (range 1) } \\
\text { (s) }\end{array}$ & $\begin{array}{r}\text { Exposure time (range 2) } \\
(\mathrm{s})\end{array}$ \\
\hline RSS & 2457679.4 & 8 & $4 \times 150$ & $3 \times 150+4 \times 30$ \\
\hline RSS & 2457694.3 & 23 & $2 \times 150+2 \times 30$ & $2 \times 100+2 \times 30$ \\
\hline HRS & 2457679.3 & 8 & $3 \times 1200$ & $2 \times 1200$ \\
\hline HRS & 2457698.3 & 27 & $3 \times 1200$ & $2 \times 1200$ \\
\hline HRS & 2457703.3 & 32 & $3 \times 1200$ & $2 \times 1200$ \\
\hline HRS & 2457716.3 & 45 & $3 \times 1200$ & $2 \times 1200$ \\
\hline HRS & 2457733.2 & 62 & $3 \times 1200$ & $2 \times 1200$ \\
\hline Spectropol & 2457686.3 & 15 & $8 \times 120$ & $8 \times 120+4 \times 60$ \\
\hline Spectropol & 2457714.3 & 43 & $8 \times 120$ & $8 \times 120+4 \times 60$ \\
\hline
\end{tabular}


Table A9. Line IDs, EWs, FWHMs, and fluxes of some emission lines in the nova SMCN 2016-10a spectra.

\begin{tabular}{|c|c|c|c|c|c|}
\hline Line & $\lambda_{0}$ & $(\AA){ }^{\operatorname{EW}(\lambda)}$ & $\begin{array}{r}\text { FWHM } \\
\left(\mathrm{km} \mathrm{s}^{-1}\right)\end{array}$ & $\begin{array}{r}\text { Flux } \\
\mathrm{erg} \mathrm{cm}^{-2} \mathrm{~s}^{-1}\end{array}$ & $\left(t-t_{0}\right)$ \\
\hline$[\mathrm{Ne} \mathrm{V}]$ & 3426 & - & - & - & 32 \\
\hline $\mathrm{H} \eta$ & 3835 & - & - & - & 8 \\
\hline $\mathrm{H} \zeta$ & 3889 & $-11 \pm 2$ & $2250 \pm 100$ & - & 8 \\
\hline $\mathrm{H} \epsilon$ & 3970 & $-15 \pm 10$ & $2300 \pm 100$ & $1.54 \times 10^{-12}$ & 8 \\
\hline $\mathrm{H} \delta$ & 4102 & $-85 \pm 10$ & $3300 \pm 100$ & $2.24 \times 10^{-12}$ & 8 \\
\hline $\mathrm{H} \gamma$ & 4341 & $-100 \pm 10$ & $3450 \pm 100$ & $2.48 \times 10^{-12}$ & 8 \\
\hline $\mathrm{He}$ I & 4388 & - & - & - & 62 \\
\hline $\mathrm{N}$ III & 4638 & - & - & - & 8 \\
\hline He II & 4686 & _ & _ & _- & 8 \\
\hline $\mathrm{H} \beta$ & 4861 & $-300 \pm 20$ & $3500 \pm 100$ & $4.73 \times 10^{-12}$ & 8 \\
\hline$[\mathrm{O}$ III $]$ & 4959 & - & - & - & 20 \\
\hline $\mathrm{N}$ II & 5001 & - & - & - & 5 \\
\hline [O III] & 5007 & $-290 \pm 20$ & $3000 \pm 100$ & $1.59 \times 10^{-13}$ & 20 \\
\hline He I & 5016 & - & - & - & 5 \\
\hline Fe II & 5018 & - & - & - & 5 \\
\hline Fe II & 5169 & - & - & - & 5 \\
\hline $\mathrm{N}_{\text {II }}$ & 5679 & - & - & - & 8 \\
\hline$[\mathrm{N}$ II $]$ & 5755 & - & - & - & 8 \\
\hline He I & 5876 & - & - & - & 8 \\
\hline $\mathrm{Na}$ I D & 5889 & - & - & - & 8 \\
\hline $\mathrm{N}$ II & 5938 & - & - & - & 8 \\
\hline $\mathrm{H} \alpha$ & 6563 & $-1500 \pm 100$ & $3500 \pm 100$ & $1.82 \times 10^{-11}$ & 8 \\
\hline $\mathrm{N}_{\mathrm{I}}$ & 7452 & - & - & - & 8 \\
\hline O IV & 7713 & - & - & - & 62 \\
\hline$[\mathrm{C}$ IV $]$ & 7726 & - & - & - & 62 \\
\hline $\mathrm{OI}$ & 7773 & $-135 \pm 10$ & $2400 \pm 100$ & $1.83 \times 10^{-12}$ & 8 \\
\hline Mg II & 7896 & - & - & - & 8 \\
\hline $\mathrm{N}_{\mathrm{I}}$ & 8212 & - & - & - & 8 \\
\hline Mg II & 8232 & - & - & - & 8 \\
\hline $\mathrm{O}_{\mathrm{I}}$ & 8446 & $-750 \pm 50$ & $3000 \pm 100$ & $4.00 \times 10^{-12}$ & 8 \\
\hline $\mathrm{N}_{\mathrm{I}}$ & 8617 & - & - & - & 8 \\
\hline $\mathrm{N}_{\mathrm{I}}$ & 8692 & - & - & - & 8 \\
\hline
\end{tabular}

